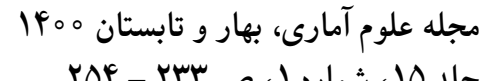

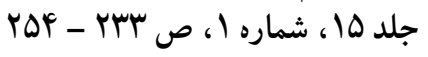

DOI: $10.29252 /$ jss.15.1.233

مقاله بزوهشى

تحليل وابستكى دو متغيره با استفاده از اندازههاى واگر ايى جفرى و هلينجر براساس برآورد

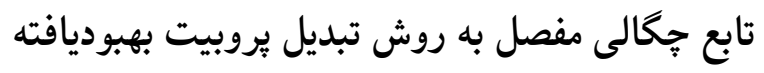

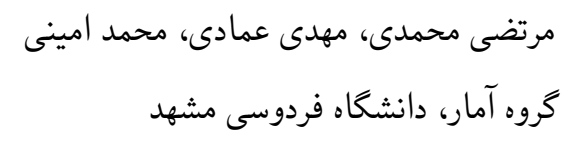

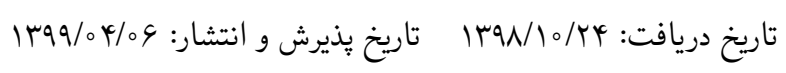

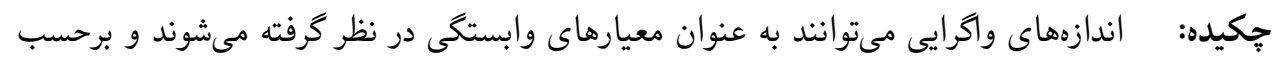

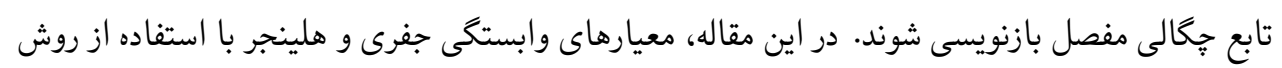

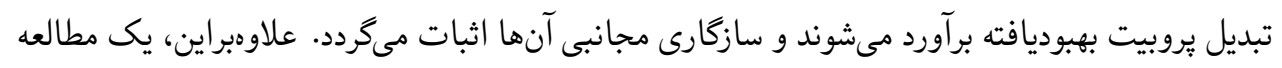

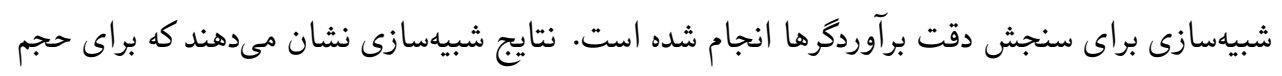

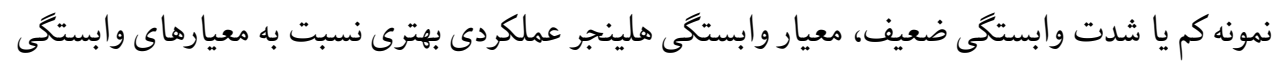

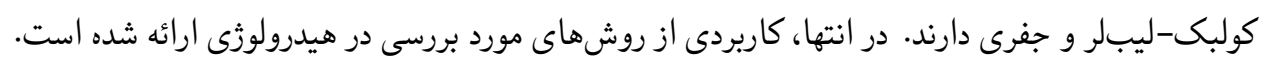

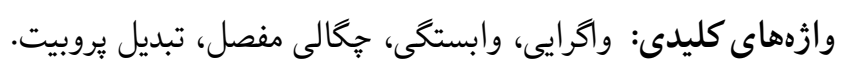

| ا

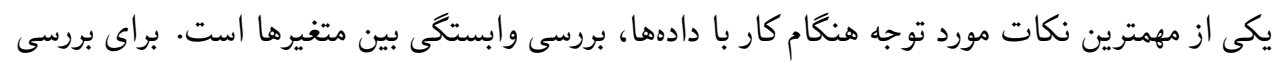

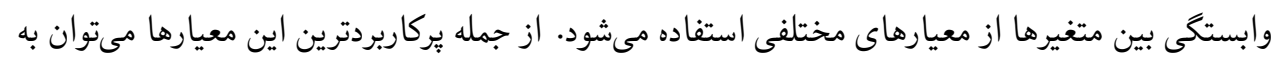

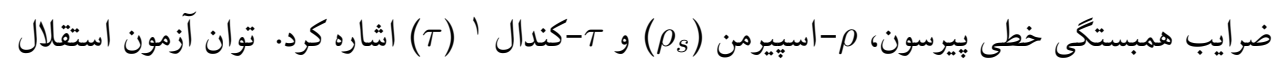

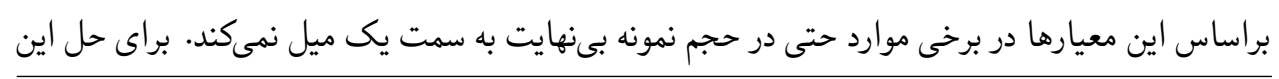

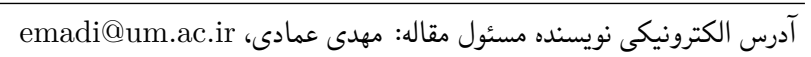

${ }^{1}$ Kendall's Tau

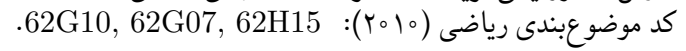




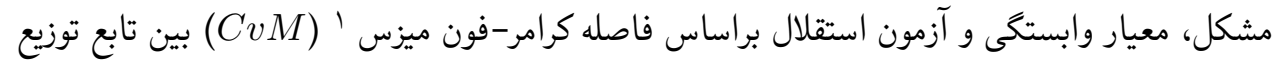

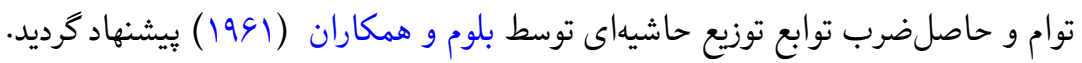

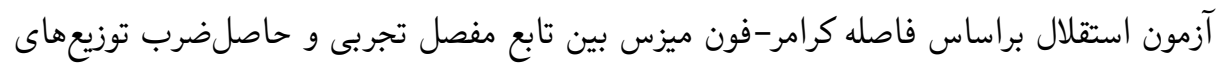

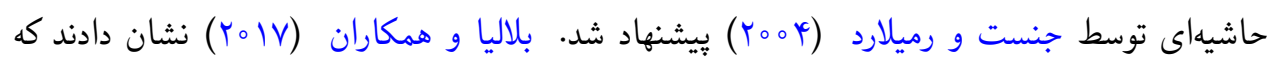

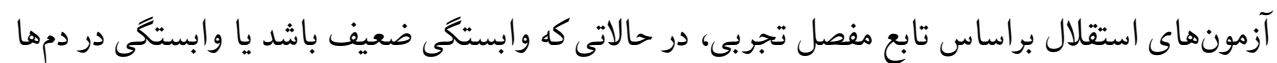

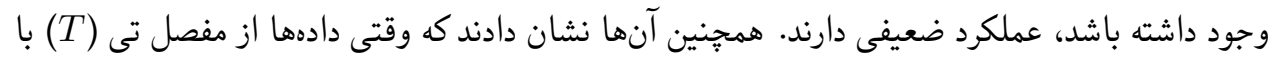

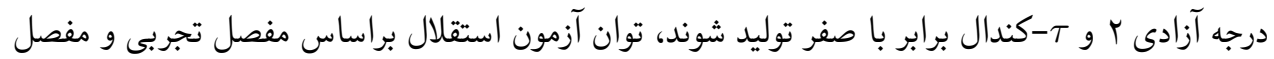

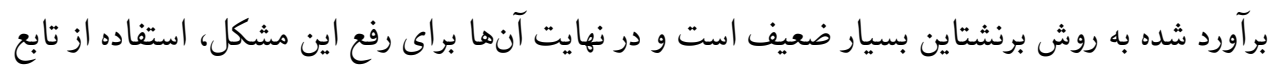

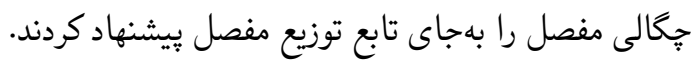

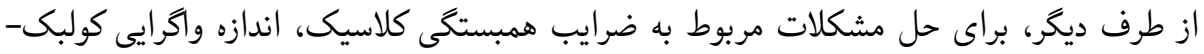

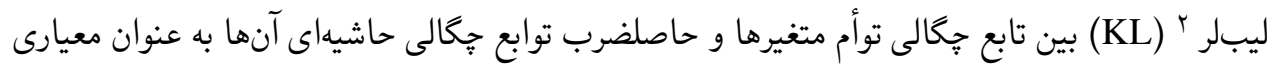

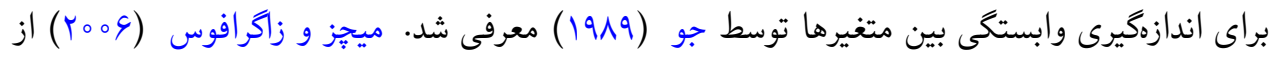

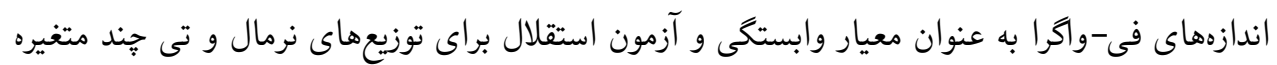

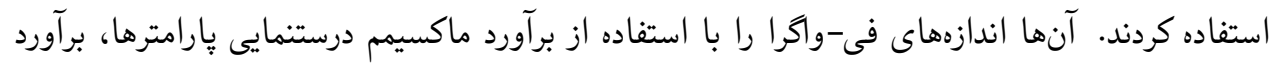

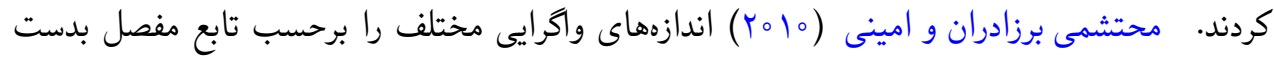

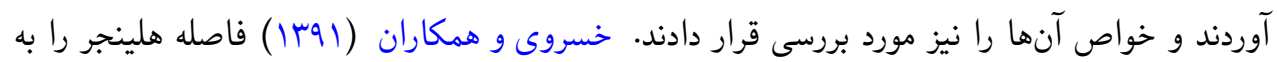

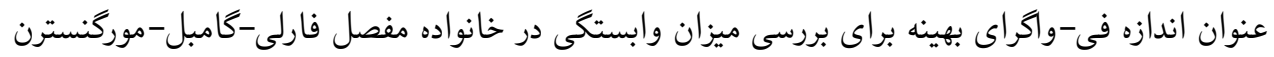

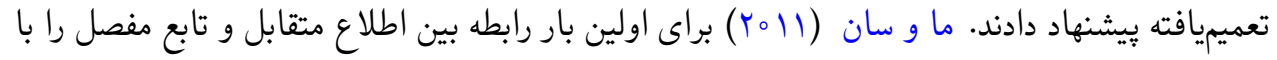

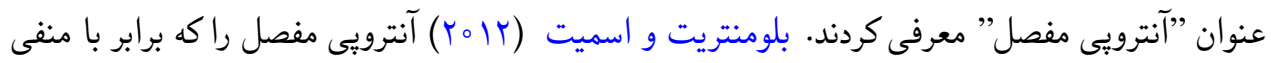

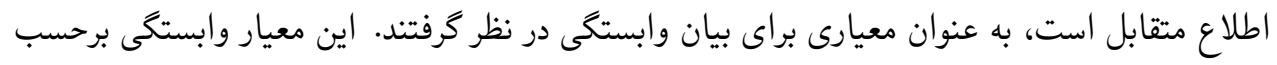

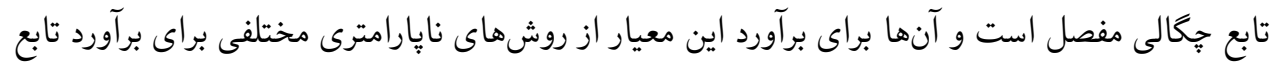

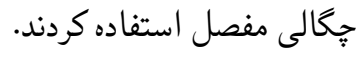

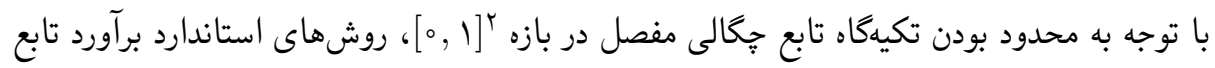

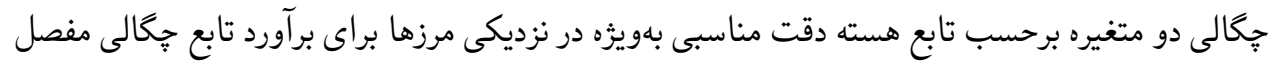

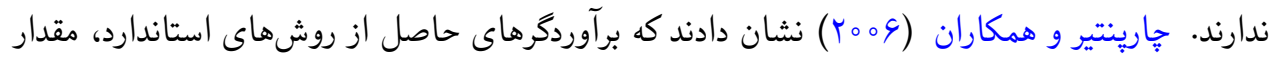

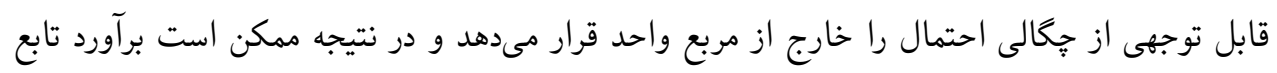

${ }^{1}$ Cramer-von Mises

${ }^{2}$ Kullback-Leibler 


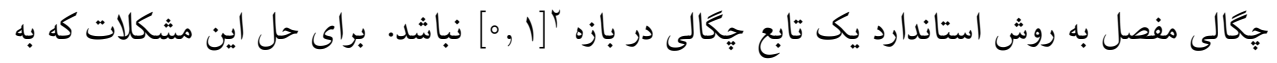

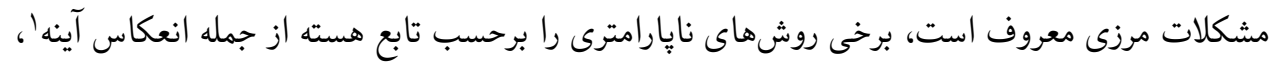

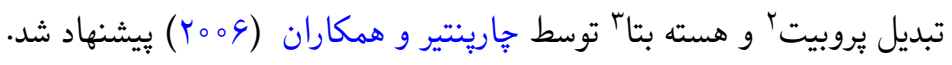

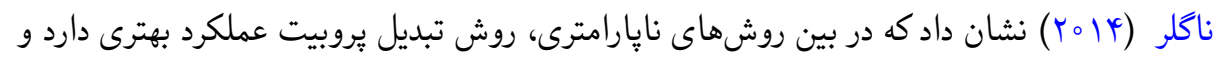

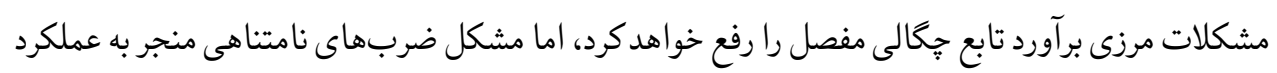

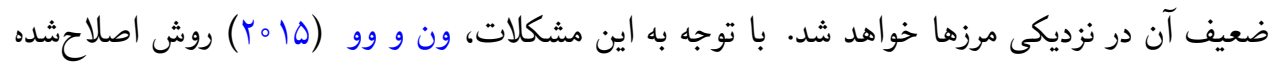

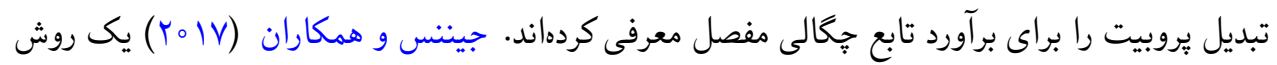

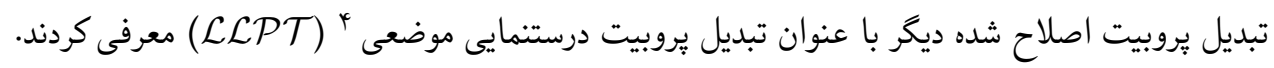

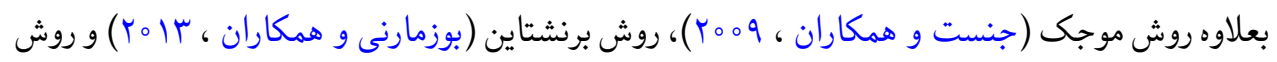

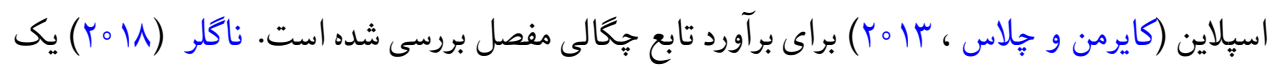

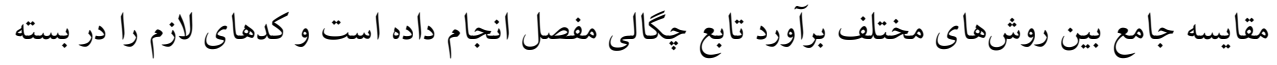
kdecopula

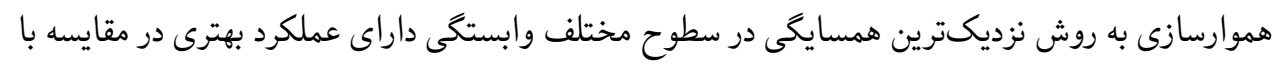
ساير روشهاى مورد بررسى است.

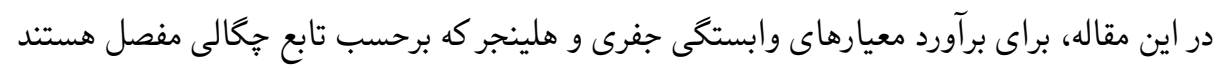

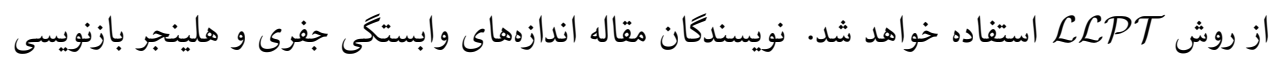

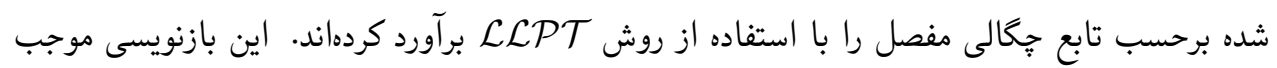

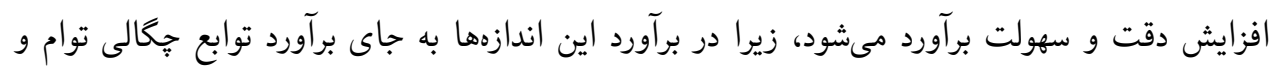

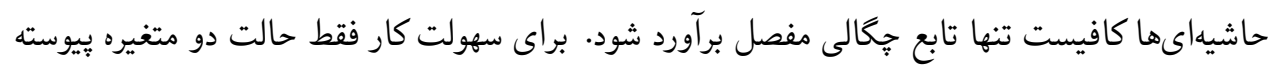

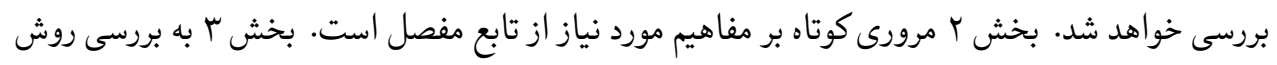

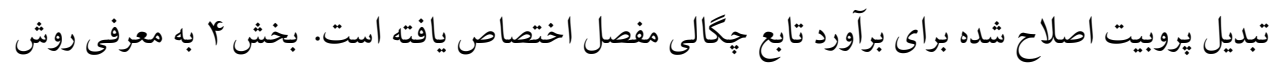

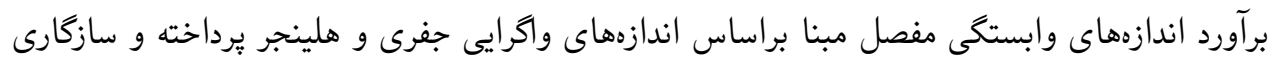

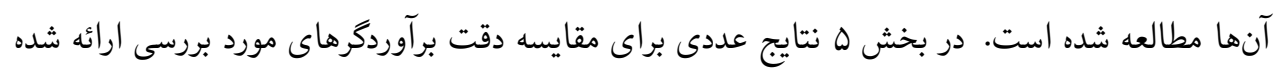

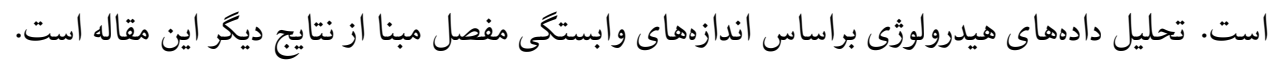

${ }^{1}$ Mirror-reflection

${ }^{2}$ Probit transformation

${ }^{3}$ Beta kernel

${ }^{4}$ Local Likelihood Probit Transformation 


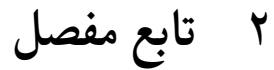

تابع مفصل ارتباط بين توزيع جند متغيره و توزيعهاى حاشيهاى يك متغيره را بيان مىكند (اسكلار ،

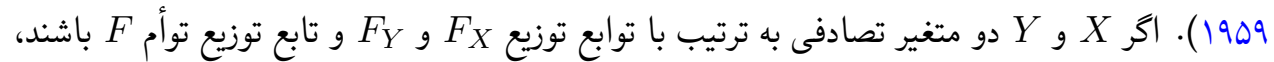
آنكاه تابع مفصل C و وجود دارد به طورىكه

$$
F(x, y)=C\left(F_{X}(x), F_{Y}(y)\right), \quad x, y \in \mathbb{R} .
$$

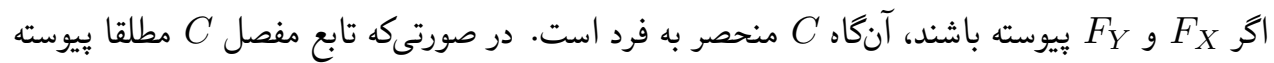

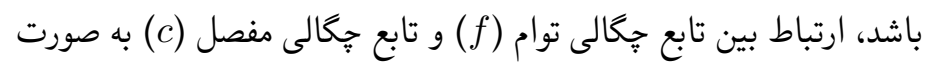

$$
f(x, y)=c\left(F_{X}(x), F_{Y}(y)\right) f_{X}(x) f_{Y}(y), \quad x, y \in \mathbb{R},
$$

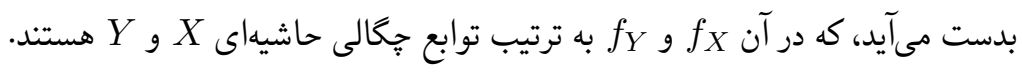

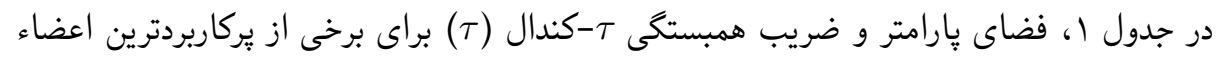

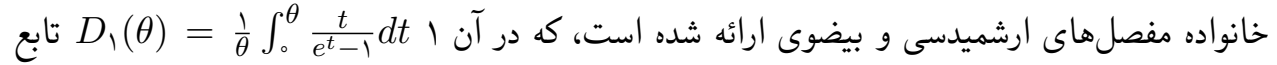

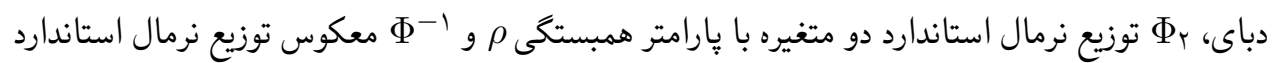

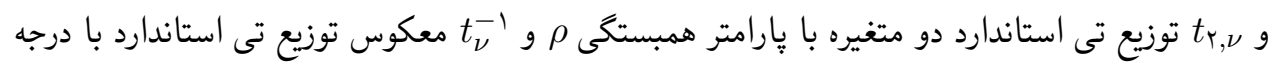

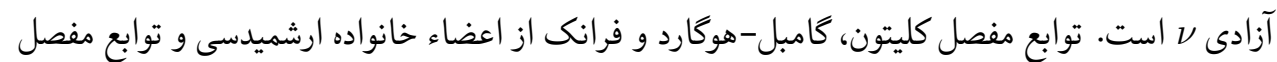

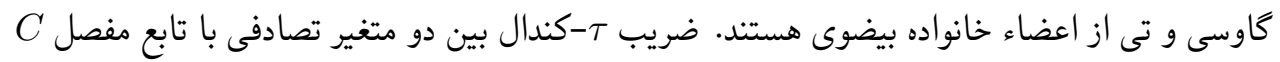

\begin{tabular}{|c|c|c|c|}
\hline 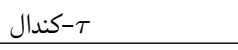 & فضاى يارامتر & $C(u, v)$ & مفصل \\
\hline$\frac{\theta}{\theta+r}$ & $\theta \in(-1,+\infty)-\{\circ\}$ & $\left(u^{-\theta}+v^{-\theta}-1\right)^{-1 / \theta}$ & 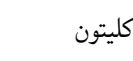 \\
\hline$\frac{\theta-1}{\theta}$ & $\theta \in[1,+\infty)$ & $\exp \left\{-\left[(-\ln u)^{\theta}+(-\ln v)^{\theta}\right]^{1 / \theta}\right\}$ & 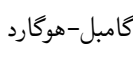 \\
\hline $1+\frac{\leftarrow}{\theta}\left(D_{1}(\theta)-1\right)$ & $\theta \in(-\infty,+\infty)-\{0\}$ & $\frac{-1}{\theta} \log \left\{1+\frac{\left(e^{-u \theta}-1\right)\left(e^{-v \theta}-1\right)}{e^{-\theta}-1}\right\}$ & 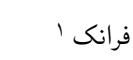 \\
\hline$\frac{r}{\pi} \arcsin (\rho)$ & $\rho \in[-1,+1]$ & $\Phi_{\curlyvee}\left(\Phi^{-1}(u), \Phi^{-1}(v) ; \rho\right)$ & 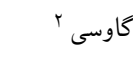 \\
\hline$\frac{r}{\pi} \arcsin (\theta)$ & $\rho \in[-1,+1], \nu>1$ & $t_{\curlyvee, \nu}\left(t_{\nu}^{-1}(u), t_{\nu}^{-1}(v) ; \rho\right)$ & ت \\
\hline
\end{tabular}

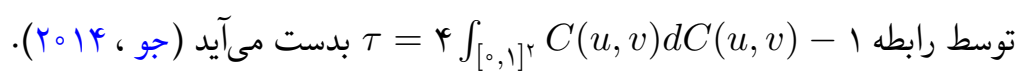




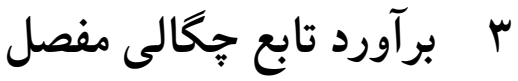

فرض كنيد

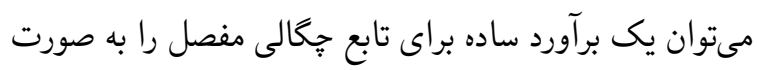

$$
\hat{c}_{n}(u, v)=\frac{1}{n} \sum_{i=1}^{n} K_{b_{n}}\left(u-\tilde{U}_{i}\right) K_{b_{n}}\left(v-\tilde{V}_{i}\right), \quad(u, v) \in[\circ, 1]^{\top},
$$

در نظر كرفت كه در آن (·) مقادير (n+1)

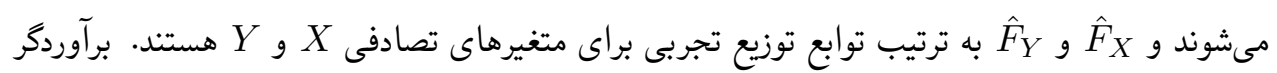

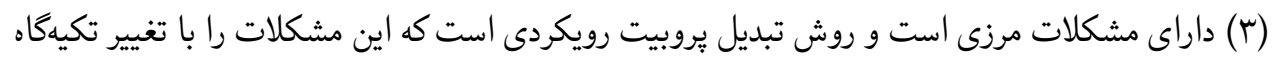

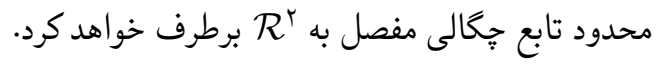

فرض كنيد بردار

از تبديل بروبيت روى بردار شبه مشاهدات به صورت

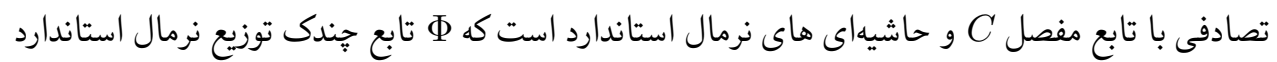

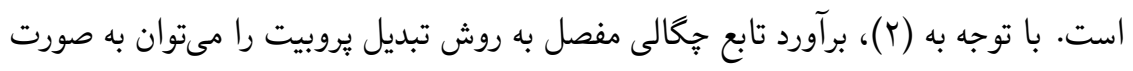

$$
\hat{c}_{n}^{(\mathcal{P T})}(u, v)=\frac{\hat{f}_{n}\left(\Phi^{-1}(u), \Phi^{-1}(v)\right)}{\phi\left(\Phi^{-1}(u)\right) \phi\left(\Phi^{-1}(v)\right)}, \quad(u, v) \in(\circ, 1)^{\Upsilon},
$$

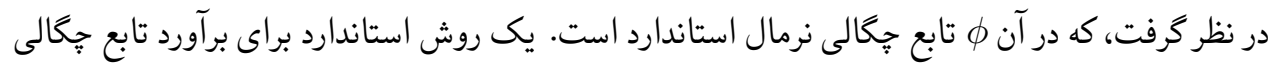
دو متغيره

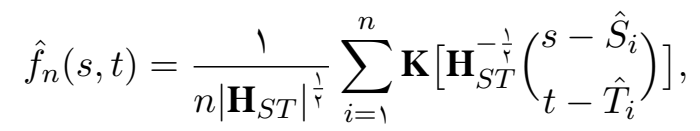

نوشت، كه در آن

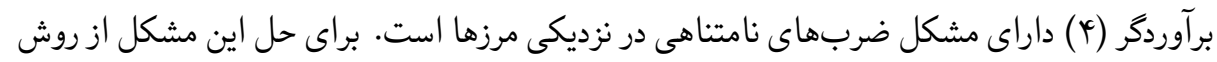

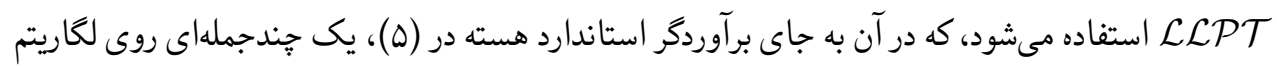




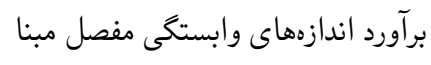

rTh

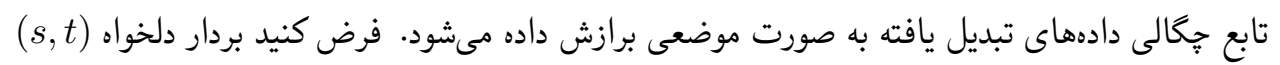

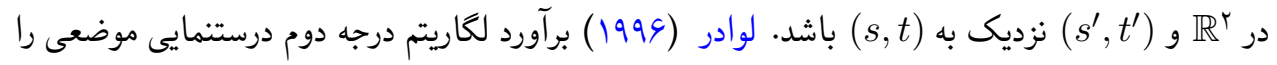
براساس شبه نمونه تبديل شده

$$
\begin{aligned}
& \log f\left(s^{\prime}, t^{\prime}\right)=a_{\Upsilon, o}(s, t)+a_{\curlyvee, 1}(s, t)\left(s^{\prime}-s\right)+a_{\Upsilon, Y}(s, t)\left(t^{\prime}-t\right) \\
& +a_{\curlyvee, \Upsilon}(s, t)\left(s^{\prime}-s\right)^{\Upsilon}+a_{\curlyvee, \digamma}(s, t)\left(t^{\prime}-t\right)^{\Upsilon}+a_{\Upsilon, \Delta}(s, t)\left(s^{\prime}-s\right)\left(t^{\prime}-t\right) \\
& \equiv P_{a_{\uparrow}}\left(s^{\prime}-s, t^{\prime}-t\right)
\end{aligned}
$$

تعريف كرد، كه در آن بردار (a

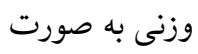

$$
\begin{aligned}
& \hat{a}_{\curlyvee}(s, t)=\arg \max _{a_{\digamma}}\left\{\sum_{i=1}^{n} \mathbf{K}\left(\mathbf{H}_{S T}^{-\frac{1}{\grave{T}}}\left(\begin{array}{c}
s-\hat{S}_{i} \\
t-\hat{T}_{i}
\end{array}\right)\right) P_{a_{\digamma}}\left(\hat{S}_{i}-s, \hat{T}_{i}-t\right)\right.
\end{aligned}
$$

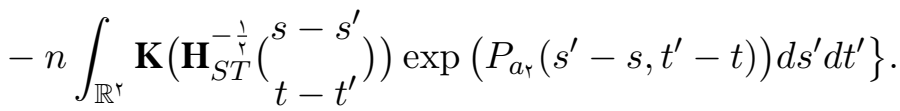

حاصل مىشود. بنابراين برآورد تابع f(s, برابر با

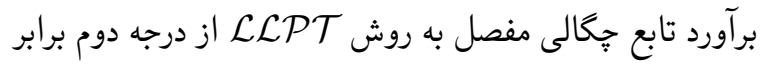

$$
\hat{c}_{n}^{(\mathcal{L L P T})}(u, v)=\frac{\tilde{f}^{p}\left(\Phi^{-1}(u), \Phi^{-1}(v)\right)}{\phi\left(\Phi^{-1}(u)\right) \phi\left(\Phi^{-1}(v)\right)}, \quad(u, v) \in[\circ, 1]^{\Upsilon} .
$$

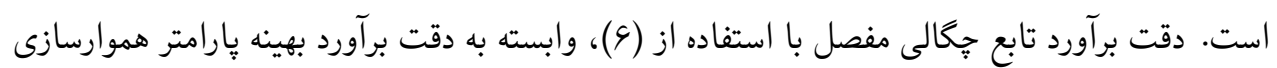

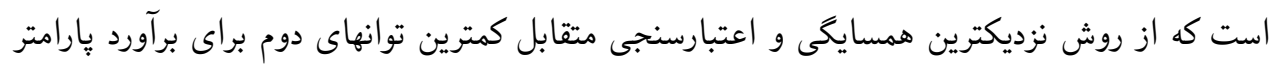

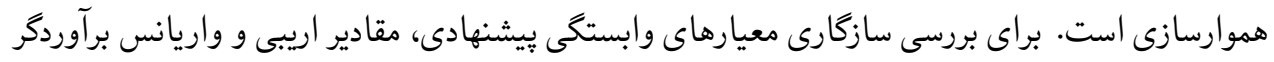




$$
\begin{aligned}
& \text { (Y.IV ، مورد نياز است كه به صورت (جينس و همكاران ) } \\
& \operatorname{Bias}\left(\hat{c}_{n}^{(\mathcal{L} \mathcal{L P T})}(u, v)\right)=-\frac{b_{n}^{\kappa}}{\wedge} \frac{c(u, v)}{\phi\left(\Phi^{-1}(u)\right) \phi\left(\Phi^{-1}(v)\right)}\left\{\frac{\partial^{\kappa} g}{\partial x^{\kappa}}+\frac{\partial^{\kappa} g}{\partial y^{\kappa}}+r \frac{\partial^{\kappa} g}{\partial x^{r} \partial y^{r}}\right. \\
& \left.+\uparrow\left(\frac{\partial^{r} g}{\partial x^{r}} \frac{\partial g}{\partial x}+\frac{\partial^{r} g}{\partial y^{r}} \frac{\partial g}{\partial y}+\frac{\partial^{r} g}{\partial x^{\ulcorner} \partial y} \frac{\partial g}{\partial y}+\frac{\partial^{r} g}{\partial x \partial y^{r}} \frac{\partial g}{\partial x}\right)\right\}(x, y)+o\left(b_{n}^{\leftarrow}\right), \\
& \operatorname{Var}\left(\hat{c}_{n}^{(\mathcal{L} \mathcal{L P} \mathcal{T})}(u, v)\right)=\frac{\diamond}{\wedge \pi n b_{n}^{\Upsilon}} \frac{c(u, v)}{\phi\left(\Phi^{-1}(u)\right) \phi\left(\Phi^{-1}(v)\right)}+o\left(\frac{1}{n b_{n}^{\Upsilon}}\right) . \\
& \text { است، كه در آن } \\
& . y=\Phi^{-1}(v)
\end{aligned}
$$

\section{F}

معيارهاى متفاوتى براى اندازه اندازهاى وآكرايى كولبك-ليبلر، جفرى و هلينجر براى محاسبه فاصله بين

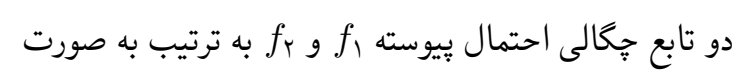

$$
\begin{aligned}
K L D\left(f_{\uparrow} \mid f_{\curlyvee}\right) & =\int_{\mathbb{R}} f_{\uparrow}(x) \log \frac{f_{\uparrow}(x)}{f_{\Upsilon}(x)} d x, \\
J D\left(f_{\uparrow} \mid f_{\Upsilon}\right) & =\int_{\mathbb{R}}\left(f_{\uparrow}(x)-f_{\curlyvee}(x)\right)\left(\log f_{\uparrow}(x)-\log f_{\Upsilon}(x)\right) d x, \\
H D\left(f_{\uparrow} \mid f_{\Upsilon}\right) & =\int_{\mathbb{R}}\left(\sqrt{f_{\uparrow}(x)}-\sqrt{f_{\Upsilon}(x)}\right)^{\Upsilon} d x .
\end{aligned}
$$

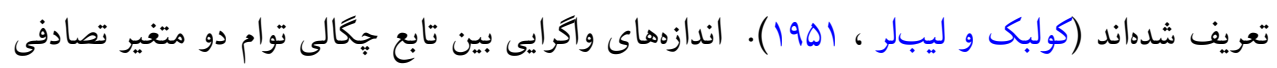

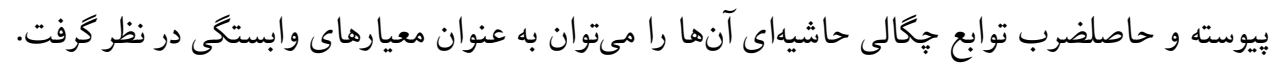

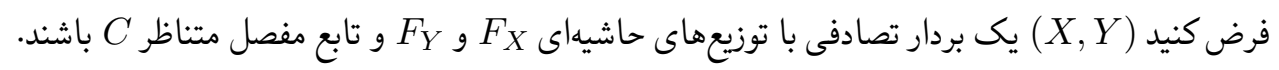


ب.... برآورد اندازهاى وابستى مفصل مبنا

YYo

$$
\begin{aligned}
& \text { با توجه به (r) و (9)، اندازه وابستخى كولبك-ليبلر مفصل مبنا توسط ما و سان (1) (Y0ب) صورت } \\
& K L D(c) \equiv K L D\left(f \mid f_{X} f_{Y}\right)=\int_{[0,1]^{r}} c(u, v) \log (c(u, v)) d u d v
\end{aligned}
$$

معرفى شد. به طور مشابه، اندازههاى وابستخى جفرى و هلينجر مفصل مبنا با استفاده از رابطه (Y) توسط

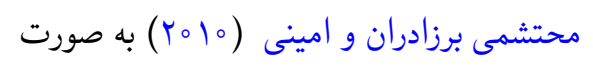

$$
\begin{aligned}
J D(c) & \equiv J D\left(f \mid f_{X} f_{Y}\right)=\int_{[\circ, 1]^{r}}(c(u, v)-1) \log (c(u, v)) d u d v \\
H D(c) & \equiv H D\left(f \mid f_{X} f_{Y}\right)=\int_{[0,1]^{\top}}(\sqrt{c(u, v)}-1)^{r} d u d v .
\end{aligned}
$$

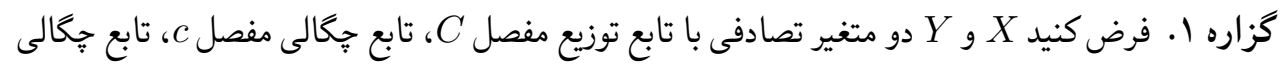

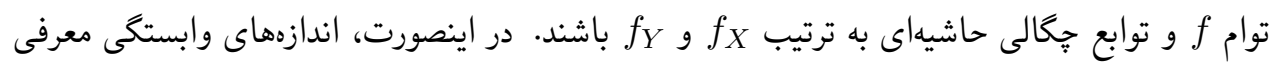

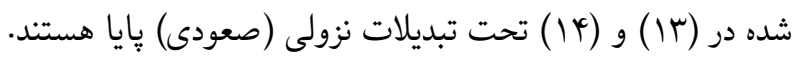

برهان: اخر م و م دو تبديل نزولى دلخواه روى متغيرهاى تصادفى X و و Y باشند، با استفاده از قضيه

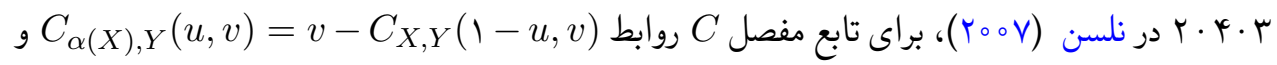
برقرار است. براى تابع جُالى مفصل $C_{\alpha(X), \beta(Y)}(u, v)=u+v-1-C_{X, Y}(1-u, 1-v)$ روابط (u, است. بنابراين

$$
\begin{aligned}
J D_{\alpha(X), Y}(c) & =\int_{[0,1]^{Y}}\left(c_{\alpha(X), Y}(u, v)-1\right) \log \left(c_{\alpha(X), Y}(u, v)\right) d u d v \\
& =\int_{[\circ, 1]^{\top}}(c(z, v)-1) \log (c(z, v)) d z d v \\
& =J D_{X, Y}(c),
\end{aligned}
$$




$$
\text { كه در آن تساوى دوم با تغيير متغير u=z - } 1 \text { برقرار است. با استلالى مشابه، }
$$

$$
\begin{aligned}
J D_{\alpha(X), \beta(Y)}(c) & =\int_{\left[{ }^{\circ}, 1\right]^{r}}\left(c_{\alpha(X), \beta(Y)}(u, v)-1\right) \log \left(c_{\alpha(X), \beta(Y)}(u, v)\right) d u d v \\
& =\int_{[0,1]^{r}}(c(z, w)-1) \log (c(z, w)) d z d w \\
& =J D_{X, Y}(c),
\end{aligned}
$$

كه در آن تساوى دوم با تغيير متغير

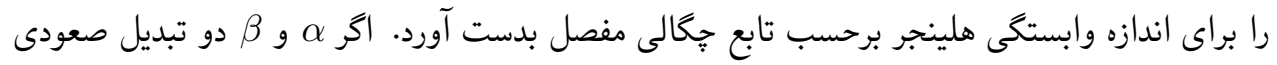

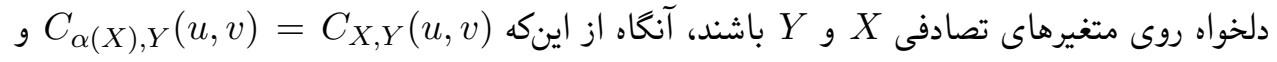

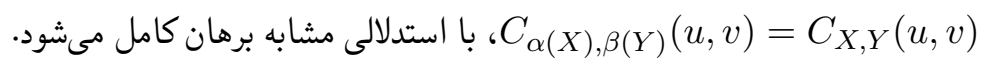

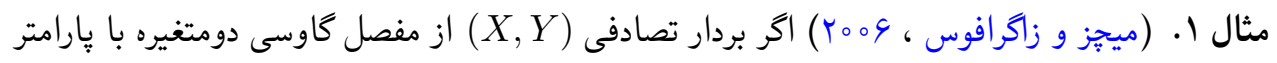
وابستخى م باشند، آنكاه

$$
\begin{aligned}
& K L D(c)=-\frac{1}{r} \log \left(1-\rho^{r}\right), \\
& J D(c)=\frac{1}{1-\rho^{r}}-1, \\
& H D(c)=r\left(1-r \frac{\left(1-\rho^{r}\right)^{\frac{1}{r}}}{\left(r-\rho^{r}\right)^{\frac{1}{r}}}\right) .
\end{aligned}
$$

اين اندازهها به ازاى ه

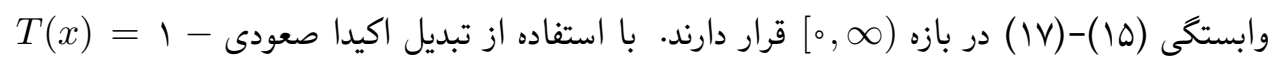
دx معيار وابستخى استاندارد كولبك-ليبلر، جفرى و هلينجر بر حسب تابع مفصل به ترتيب از نمادهاى كار امت م

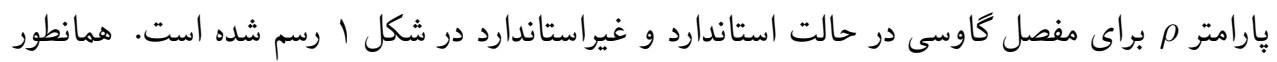

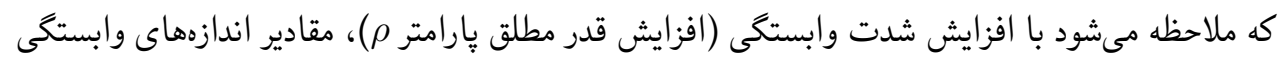

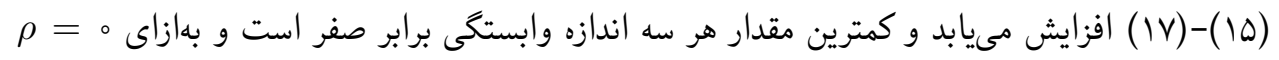




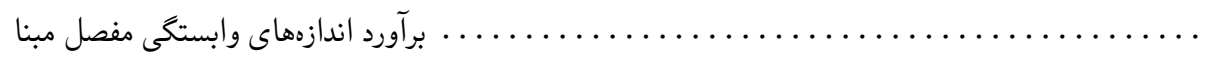

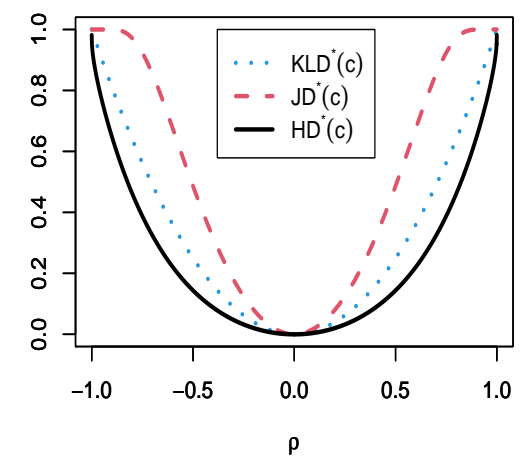

(ب)

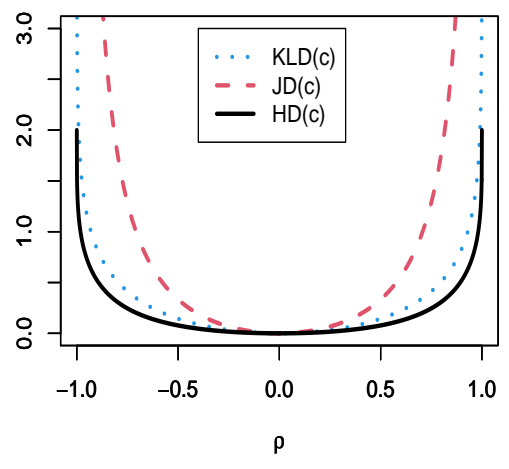

(الف)

شكل ا. اندازهاى وابستكى براى مفصل كاوسى با پارامتر م در حالت الف-غيراستاندارد و ب- استاندارد

حاصل مىشود. اندازه وابستكى هلينجر تنها انداز وابستخى در بين اندازههاى وابستكى در شكل ا است كه وابستخى خطى (1)

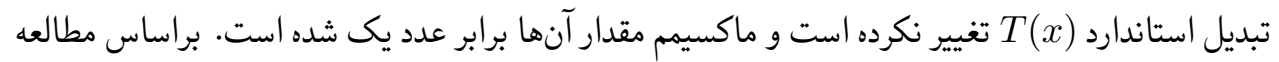

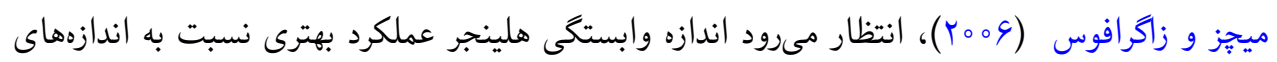
وابستكى جفرى و كولبك-ليبلر داشته باشد، زيرا مقاير كمترى نسبت به آنها اختيار مىكند و و همجنينين مقدار ماكسيم آن در حالت غيراستاندارد متناهى است.

براى نمونه تصادفى

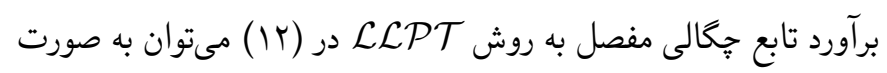

$$
\widehat{K L D}(c)=\frac{1}{n} \sum_{i=1}^{n} \log \left(\hat{c}^{(\mathcal{L L P} \mathcal{T})}\left(\tilde{U}_{i}, \tilde{V}_{i}\right)\right)
$$


ارائه كرد. به طور مشابه، برآوردكرهاى گشتاورى اندازههاى وابستگى جفرى و هلينجر با جايخذارى برآورد

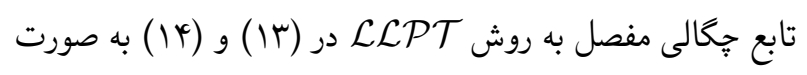

$$
\begin{aligned}
& \widehat{J D}(c)=\frac{1}{n} \sum_{i=1}^{n}\left(1-\frac{1}{\hat{c}^{(\mathcal{L L P} \mathcal{T})}\left(\tilde{U}_{i}, \tilde{V}_{i}\right)}\right) \log \left(\hat{c}^{(\mathcal{L L P} \mathcal{T})}\left(\tilde{U}_{i}, \tilde{V}_{i}\right)\right) \\
& \widehat{H D}(c)=\frac{1}{n} \sum_{i=1}^{n}\left(1-\frac{1}{\sqrt{\hat{c}^{(\mathcal{L L P} \mathcal{T})}\left(\tilde{U}_{i}, \tilde{V}_{i}\right)}}\right)^{\Upsilon}
\end{aligned}
$$

معرفى مىشوند، كه در آن

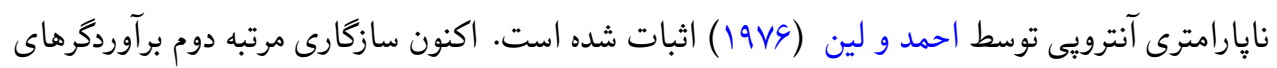

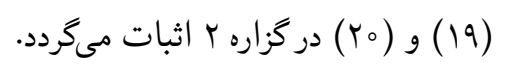

كزاره r. فرض كنيد n, ., c باشد. اخر ه $E\left(c(U, V)^{r}\right)<\infty$ الف) هنغامى كه

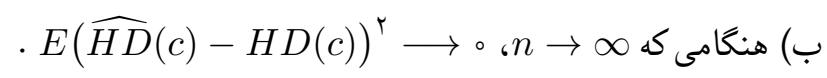

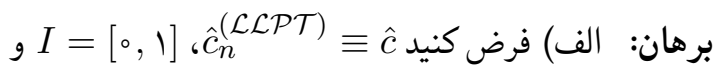

$$
\begin{aligned}
& J D(c)=\int_{I^{\dagger}}\left(1-\frac{1}{c(u, v)}\right) \log (c(u, v)) d C(u, v), \\
& \widehat{J D}(c)=\int_{I^{\Upsilon}}\left(1-\frac{1}{\hat{c}(u, v)}\right) \log (\hat{c}(u, v)) d C_{n}(u, v) \text {, } \\
& \theta(c)=\int_{I^{\Gamma}}\left(1-\frac{1}{c(u, v)}\right) \log (c(u, v)) d C_{n}(u, v) .
\end{aligned}
$$




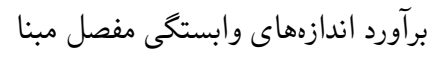

با استفاده از نامساوى مينكوفسكى مىتوان نوشت،

$$
\begin{aligned}
E(\widehat{J D}(c)-J D(c))^{\Upsilon} & =E[(\widehat{J D}(c)-\theta(c))+(\theta(c)-J D(c))]^{r} \\
& \leq\left[E^{1 / \Upsilon}(\widehat{J D}(c)-\theta(c))^{r}+E^{1 / r}(\theta(c)-J D(c))^{r}\right]^{r} \\
& =\left(P_{1}^{1 / \Upsilon}+P_{\curlyvee}^{1 / r}\right)^{r} .
\end{aligned}
$$

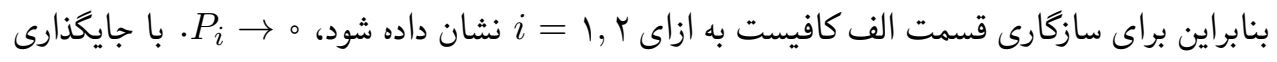

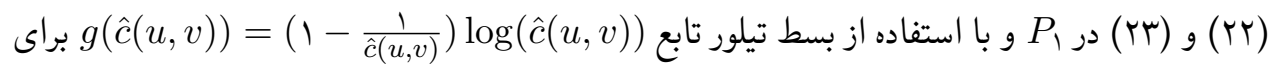
مقادير ثابت

$$
\begin{aligned}
& P_{\uparrow}=E\left[\int_{I^{\Upsilon}}\left(\left(1-\frac{1}{\hat{c}(u, v)}\right) \log (\hat{c}(u, v))-\left(1-\frac{1}{c(u, v)}\right) \log (c(u, v))\right) d C_{n}(u, v)\right]^{\Upsilon} \\
& =E\left[\int_{I^{\leftarrow}}\left(\frac{c(u, v)-1+\log (c(u, v))}{c^{\Upsilon}(u, v)}\right)(\hat{c}(u, v)-c(u, v)) d C_{n}(u, v)+o\left(b_{n}^{\leftarrow}\right)\right]^{\Upsilon} \\
& =E\left[\frac{1}{n} \sum_{i=1}^{n}\left(\frac{c\left(U_{i}, V_{i}\right)-1+\log \left(c\left(U_{i}, V_{i}\right)\right)}{c^{\curlyvee}\left(U_{i}, V_{i}\right)}\right)\left(\hat{c}\left(U_{i}, V_{i}\right)-c\left(U_{i}, V_{i}\right)\right)+o\left(b_{n}^{\leftarrow}\right)\right]^{\curlyvee} \\
& =\frac{1}{n^{\Upsilon}} E\left[\sum_{i=1}^{n}\left(\frac{c\left(U_{i}, V_{i}\right)-1+\log \left(c\left(U_{i}, V_{i}\right)\right)}{c^{\curlyvee}\left(U_{i}, V_{i}\right)}\right)^{\Upsilon}\left(\hat{c}\left(U_{i}, V_{i}\right)-c\left(U_{i}, V_{i}\right)\right)^{\Upsilon}\right] \\
& +\frac{1}{n^{\Upsilon}} E\left[\sum_{\substack{i, j=1 \\
i \neq j}}^{n}\left(\frac{c\left(U_{i}, V_{i}\right)-1+\log \left(c\left(U_{i}, V_{i}\right)\right)}{c^{\curlyvee}\left(U_{i}, V_{i}\right)}\right)\left(\frac{c\left(U_{j}, V_{j}\right)-1+\log \left(c\left(U_{j}, V_{j}\right)\right)}{c^{\curlyvee}\left(U_{j}, V_{j}\right)}\right)\right. \\
& \left.\times\left(\hat{c}\left(U_{i}, V_{i}\right)-c\left(U_{i}, V_{i}\right)\right)\left(\hat{c}\left(U_{j}, V_{j}\right)-c\left(U_{j}, V_{j}\right)\right)\right]+o\left(b_{n}^{\wedge}\right) \\
& =A_{\uparrow}+A_{\uparrow}+o\left(b_{n}^{\wedge}\right) \text {. }
\end{aligned}
$$

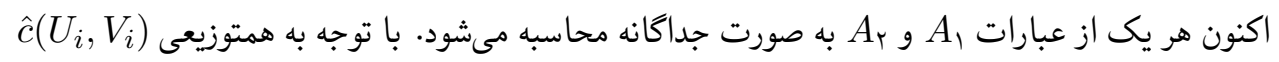




$$
\begin{aligned}
& A_{\uparrow}=\frac{1}{n} E\left[\left(\frac{c(U, V)-1+\log (c(U, V))}{c^{\curlyvee}(U, V)}\right)^{\Upsilon}(\hat{c}(U, V)-c(U, V))^{\Upsilon}\right] \\
& =\frac{1}{n} E_{(U, V)}\left\{E_{\hat{c}}\left[\left(\frac{c(U, V)-1+\log (c(U, V))}{c^{\Upsilon}(U, V)}\right)^{\Upsilon}(\hat{c}(U, V)-c(U, V))^{\Upsilon} \mid U, V\right]\right\} \\
& =\frac{1}{n} \int_{I^{\Upsilon}} E_{\hat{c}}\left[\left(\frac{c(U, V)-1+\log (c(U, V))}{c^{\Upsilon}(U, V)}\right)^{r}\right. \\
& \left.\times(\hat{c}(U, V)-c(U, V))^{\Upsilon} \mid U=u, V=v\right] c(u, v) d u d v . \\
& \text { با توجه به (V) و (^) براى هر ب) (u,v) داريم، } \\
& E_{\hat{c}}\left[\left(\frac{c(u, v)-1+\log (c(u, v))}{c^{\Upsilon}(u, v)}\right)^{\Upsilon}(\hat{c}(u, v)-c(u, v))^{\Upsilon}\right] \\
& =\left(\frac{c(u, v)-1+\log (c(u, v))}{c^{\Upsilon}(u, v)}\right)^{\Upsilon} E_{\hat{c}}(\hat{c}(u, v)-c(u, v))^{\Upsilon} \\
& =\left(\frac{c(u, v)-1+\log (c(u, v))}{c^{\Upsilon}(u, v)}\right)^{r}\left(\operatorname{Var}_{\hat{c}}(\hat{c}(u, v))+\operatorname{Bias}_{\hat{c}}^{r}(\hat{c}(u, v))\right) \\
& =\left(\frac{c(u, v)-1+\log (c(u, v))}{c^{\Upsilon}(u, v)}\right)^{\Upsilon}\left(O\left(\frac{1}{n b_{n}^{\Upsilon}}\right)+O\left(b_{n}^{\leftarrow}\right)\right) \longrightarrow \circ, \quad n \rightarrow \infty .
\end{aligned}
$$

علاوهبراين،

$$
\begin{aligned}
& E_{\hat{c}}\left[\left(\frac{c(u, v)-1+\log (c(u, v))}{c^{\Upsilon}(u, v)}\right)^{\Upsilon}(\hat{c}(u, v)-c(u, v))^{\Upsilon}\right] c(u, v) \\
& \leq\left(\frac{c(u, v)-1+\log (c(u, v))}{c^{\Upsilon}(u, v)}\right)^{\Upsilon}\left(\operatorname{Var}_{\hat{c}}(\hat{c}(u, v))+E_{\hat{c}}^{\Upsilon}(\hat{c}(u, v))+c^{\Upsilon}(u, v)\right) c(u, v) \\
& \leq\left(\frac{c(u, v)-1+\log (c(u, v))}{c^{\Upsilon}(u, v)}\right)^{\Upsilon}\left(O\left(\frac{1}{n b_{n}^{\Upsilon}}\right)+r c^{\Upsilon}(u, v)+O\left(b_{n}^{\Upsilon}\right)+c^{\Upsilon}(u, v)\right) c(u, v) \\
& \longrightarrow r c^{\Upsilon}(u, v)\left(\frac{c(u, v)-1+\log (c(u, v))}{c^{\Upsilon}(u, v)}\right)^{\Upsilon}, \quad n \rightarrow \infty .
\end{aligned}
$$

بنابراين تابع زير انتخرال \A همگرا به صفر و از بالاكراندار است. در اينصورت هنگامى كه $\rightarrow$ بـ 


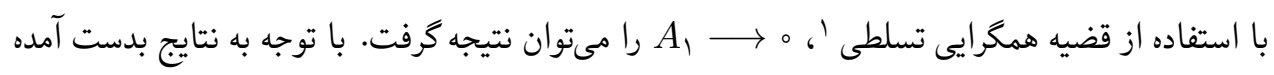

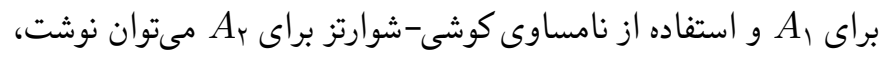

$$
A_{\uparrow} \leq \frac{1}{n^{r}} n(n-1)\left(\sqrt{A_{\uparrow}}\right)^{r} \longrightarrow \circ, \quad n \rightarrow \infty .
$$

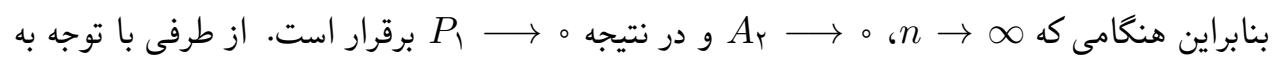

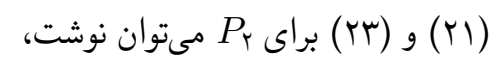

$$
\begin{aligned}
& P_{\Upsilon}=E\left(\frac{1}{n} \sum_{i=1}^{n}\left(1-\frac{1}{c\left(U_{i}, V_{i}\right)}\right) \log \left(c\left(U_{i}, V_{i}\right)\right)\right. \\
& \left.-\int_{I^{r}}\left(1-\frac{1}{c(u, v)}\right) \log c(u, v) d C(u, v)\right)^{r} \\
& =\frac{1}{n} \operatorname{Var}\left(\left(1-\frac{1}{c(U, V)}\right) \log c(U, V)\right)=O\left(\frac{1}{n}\right) \text {. } \\
& \text { در نتيجه هنكامى كه م } \\
& \text { ب) اثبات اين قسمت به روشى مشابه اثبات قسمت الف قابل ارائه است. }
\end{aligned}
$$

\section{ه - n مطالعه شبيهسازى}

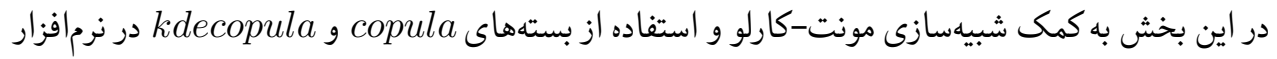

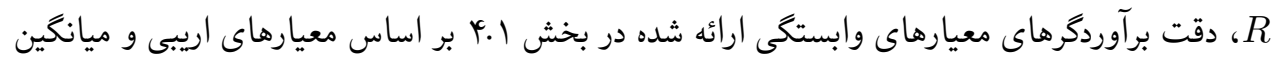

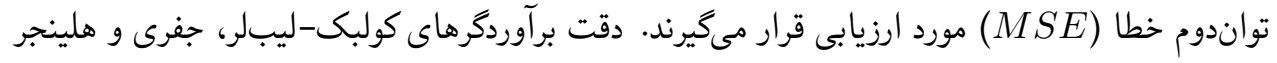

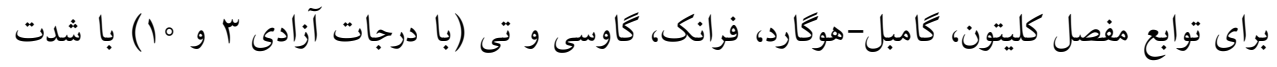

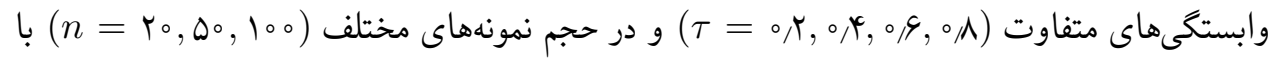

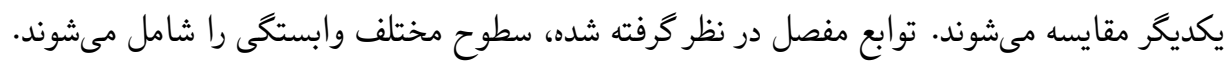

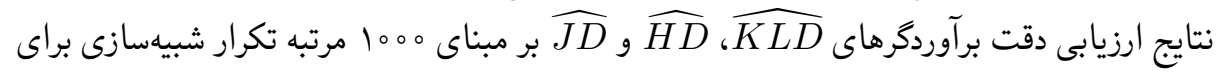

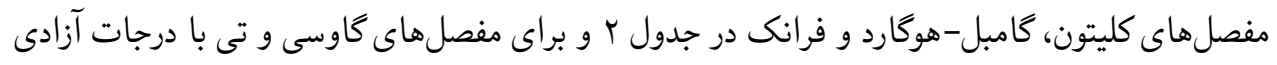

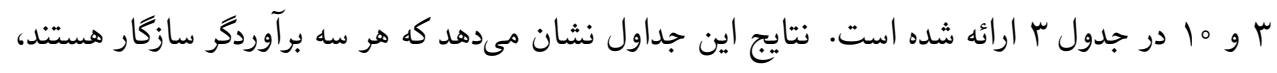


جدول r. اريبى و MSE اندازهاى وابستخى براى مفصلهاى كليتون، كامبل-هوكارد و فرانك

\begin{tabular}{|c|c|c|c|c|c|c|c|c|c|c|}
\hline \multicolumn{4}{|c|}{ MSE } & \multicolumn{4}{|c|}{ اريبي } & \multirow[b]{2}{*}{ برآوردكر } & \multirow[b]{2}{*}{$n$} & \multirow[b]{2}{*}{ مفصل } \\
\hline$\tau=\mathrm{o}, \mathrm{A}$ & $\tau=0, \bar{q}$ & $\tau=0, \mu$ & $\tau=0, \pi$ & $\tau=$ 。从 & $\tau=0,9$ & $\tau=0, \mu$ & $\tau=\circ, \bar{T}$ & & & \\
\hline$\% 401$ & $\% \% \triangle \wedge$ & 0,0 YAV & $0,0 Y Y q$ & $0,094 \mathrm{~V}$ & & 0 & 0,1490 & $\widehat{K L D}$ & & \\
\hline 0,0194 & 00144 & 0,0०AF & $000 \mathrm{VI}$ & 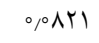 & $0 \% 4 Y q$ & $\%$ \%OI & $\% \Delta$ FD & $\widehat{H D}$ & ro & \\
\hline$T \times 490$ & 1,994T & OpYIT & 0,0179 & $-\Delta, \Delta \Delta \mid \varphi$ & $-1,0911$ & $-0, \pi T \Delta \Delta$ & -o,0NTH & $\overline{J D}$ & & \\
\hline \% TAY & $00 \times 49$ & 0,0199 & 0,0109 & ו & 0,0900 & $0 \wedge \Delta M^{\prime}$ & V0,099 & $\widehat{K L D}$ & & كليتون \\
\hline 0,0191 & 00109 & $0,0 \circ \Delta \Delta$ & 0,0044 & 0,0111 & 0 & . & $0,0 \psi_{0} \wedge$ & $\widehat{H D}$ & هo & \\
\hline r,1909 & 1,N904 & $0,10 \lambda 1$ & $\circ \wedge \Delta . \circ$ & $-r_{\lambda} \wedge V_{0}$ & $-1, \pi y \Delta V$ & $-0,4940$ & $-0 \% \Delta 90$ & $\overline{J D}$ & & \\
\hline 0,0144 & 00111 & opova & $0,00 \Delta \Delta$ & $-0,00 T_{0}$ & 0,0 Y o & $\%$ \% V V & $0,0 \Delta \Delta 9$ & $\widehat{K L D}$ & & \\
\hline 00109 & 0,0091 & $0,00 K Y$ & 0,0019 & 0,0091 & $\%$ ००० & $0 \%$ \% & 0,0419 & $\widehat{H D}$ & 100 & \\
\hline r.0919 & $1,90 \mathrm{rq}$ & 0,0909 & 0,0041 & $-19 \times 19$ & $-1, / \Delta \wedge \hat{F}$ & $-0, K Y Y$ & $-0,0$ YVG & $\widehat{J D}$ & & \\
\hline$\% \Delta \Delta Y$ & $\%$ \% q & 0,0411 & O,OTYY & 0 ollyg & 0,1599 & $0,14 \varphi_{0}$ & $0,10 \% A$ & $\widehat{K L D}$ & & \\
\hline $0 \% 419$ & $\% 179$ & $0,01 T_{0}$ & $0 \% 0 \mathrm{VV}$ & 0,1009 & $0,09 A T$ & $\% \Delta 9 \%$ & $00 \Delta 9 \Delta$ & $\widehat{H D}$ & $r_{0}$ & \\
\hline 1,4949 & oNVAr & opror & o,oTIV & $-r / F \forall \Delta r$ & - оNTAT & $-0,1 k 94$ & $-0,0 \wedge \mid Q$ & $\widehat{J D}$ & & \\
\hline $0,0 \% 09$ & $\%$ \% V & oprly & 0,0190 & OONGY & $0,0 N k y$ & 0.941 & $0,10 \mathrm{rr}$ & $\widehat{K L D}$ & & كامبل. \\
\hline 00491 & $001 \pi t$ & $\% 0 \mathrm{VA}$ & 0,0049 & 0,0940 & $0,04 Y S$ & 00094 & 0,0444 & $\widehat{H D}$ & ఎ. & \\
\hline 1,1949 & $\circ N \circ D F$ & 0,0491 & $0 \% \circ \wedge \Delta$ & $-T, \times 911$ & $-0,1019$ & $-0,1491$ & $-0,0 D / F$ & $\widehat{J D}$ & & \\
\hline 00170 & $0 \% 109$ & $0,0 \circ 1$ & $0,00 \Delta 9$ & 0,0 HOF & 0,0 א & 0,0009 & $0 \% \Delta V V$ & $\widehat{K L D}$ & & \\
\hline 00194 & r & $0,0 \circ \Lambda^{\circ}$ & $0,00 \mathrm{KY}$ & $00 \Delta 9 T$ & 0,0YMl & $0 \%$ YY & $0,04 \Delta 1$ & $\widehat{H D}$ & 100 & \\
\hline 09109 & OATMA & 0,0TYq & $0,0 \% \mu r$ & $-1, \mid 494$ & $-0,99 \times 9$ & -OMTKY & $-0,04 \circ 9$ & $\widehat{J D}$ & & \\
\hline o, KFY & $\% 0499$ & 0,0 YA & $0,0 \%$ O & 0 MlQF & $0,1 T 01$ & 0 0 149 & $0,1 \Delta \% 4$ & $\widehat{K L D}$ & & \\
\hline ו וTrו & $\% 197$ & $0,01 \% q$ & $0,0 \circ \mathrm{VV}$ & $0,1 \circ \mathrm{VI}$ & \%०AGY & $0090 \mathrm{~V}$ & 0,0901 & $\widehat{H D}$ & ro & \\
\hline WDSF & $\circ \Delta 4 \wedge \Delta$ & 0,0TYq & $0, \% \circ D$ & $-9,0 \Delta Y$ & - NTIS & $-0,1009$ & $-0 \% \mathrm{~V}$ & $\overrightarrow{J D}$ & & \\
\hline $00 \times 90$ & $0,0 \% 01$ & 0,0191 & 0,0109 & $0,0 \wedge V V$ & $0090 \%$ & 0,1014 & $0,10 \%$ & $\widehat{K L D}$ & & فرانك \\
\hline $001 T \Delta$ & $0009 V$ & 0,0091 & $0,0 \% \Delta$ & $0,0 \wedge 01$ & $0 \% \vee \Delta \wedge$ & $\% \Delta d r$ & $0,0 \mathrm{KHT}$ & $\bar{H} \bar{D}$ & $\omega_{0}$ & \\
\hline 09109 & odrav & 。orlo & $0,0 \circ \mathrm{Vq}$ & -4, N991 & $-\circ, N \circ Y_{0}$ & $-0,090 \mathrm{~V}$ & $0 \% \Delta 1 A$ & $\widehat{J D}$ & & \\
\hline 00091 & $\% 0 \wedge A F$ & $\% \circ \mathrm{Vrr}$ & $0,00 \Delta Y$ & OPYYY & 0,0499 & $\% \Delta \Delta F^{4}$ & $00 \Delta 94$ & $\widehat{K L D}$ & & \\
\hline & 0,000 & & & rוVוק & 0,0940 & $0<k y$ & & $\widehat{H D}$ & 100 & \\
\hline $0, N 4{ }^{2}$ & $0, \kappa_{0} \varphi_{0}$ & 0,0 YIF & ג & - TAYAI & $-0, \Delta V \backslash \varphi$ & $-0, \wedge \wedge 1$ & $-0 \% Y_{0} \mathrm{Y}$ & $\widehat{J D}$ & & \\
\hline
\end{tabular}

زيرا براى تمامى مفصلهاى در نظر گرفته شده، با افزايش حجم نمونه مقادير اريبى و MSE براى هاى هر سه

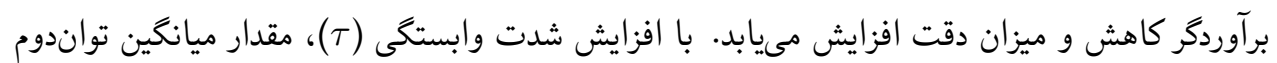

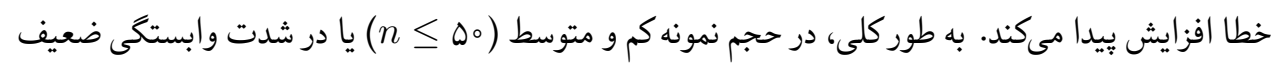

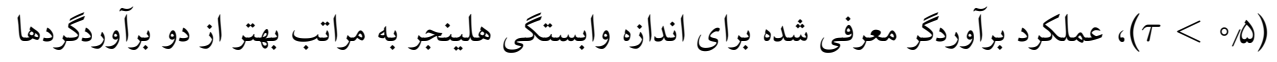

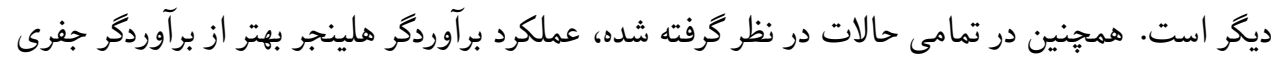

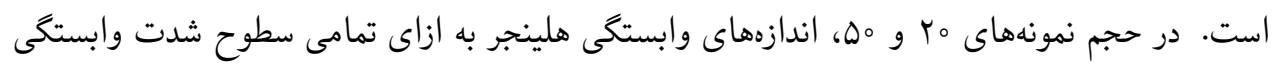




\begin{tabular}{|c|c|c|c|c|c|c|c|c|c|c|}
\hline \multirow[b]{2}{*}{$\tau=0, \wedge$} & \multicolumn{3}{|c|}{ MSE } & \multicolumn{4}{|c|}{ اريبي } & \multirow[b]{2}{*}{ برآوردكر } & \multirow[b]{2}{*}{$n$} & \multirow[b]{2}{*}{ مفصل } \\
\hline & $\tau=0, \S$ & $\tau=0,4$ & $\tau=0, \pi$ & $\tau=\circ \wedge$ & $\tau=0, q$ & $\tau=0,4$ & $\tau=0, \pi$ & & & \\
\hline$\% 0 \Delta 04$ & 0,0411 & 0,0490 & 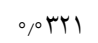 & & $0,1 \% \circ \Delta$ & 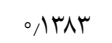 & $0,01 \%$ & $\widehat{K L D}$ & & \\
\hline$\%$ \% YYV & ס ק rा & $\circ, 0 \circ \Delta$ & $0,009 \mathrm{~V}$ & о Mाr & $0,0 k 4 t$ & 0,0 fir & $\% 0 \Delta \%$ & $\widehat{H D}$ & ro & \\
\hline rMTS4 & $1, N+19$ & $0,09 \mathrm{~W}$ & OOYOY & $-T, Y \mid Q Y$ & $-1, \pi \circ 0 \%$ & $-0,19 T V$ & $-0,09 \pi T$ & $\widehat{J D}$ & & \\
\hline \% rar & $0,0 Y 19$ & $0,11 \Delta$ & $\% 104$ & 10V9 & 0,0ArG & 0,0944 & 0,1011 & $\widehat{K L D}$ & & كاوسى \\
\hline$\% 199$ & 00011 & $0,00 \Delta r$ & 0,0041 & $0,0940^{\circ}$ & $0,0 Y \Delta \Delta$ & $0,0 r \Lambda F$ & $0,0 \varphi_{0} \mathrm{r}$ & $\widehat{H D}$ & $\omega_{0}$ & \\
\hline$r / 0 \Delta 99$ & 1,009 & 00094 & $\% \circ \vee V k$ & $-|, N A F|$ & -1, KAGK & $-0,1 V F q$ & $-0,0 \Delta \circ \Delta$ & $\widehat{J D}$ & & \\
\hline$\%$ & 0,0094 & $0,0 \circ V Y$ & $0 \% \Delta r$ & $0,0 y 4 \mid$ & 0,0494 & $0,0 \Delta T Y$ & $\% \circ \Delta \wedge F$ & $\widehat{K L D}$ & & \\
\hline$\% 10 \mathrm{r}$ & $\% \circ \mathrm{VV}$ & $0,004 \mathrm{Fr}$ & $0,001 \mathrm{~V}$ & 0,0914 & $0,01 V \Delta$ & $0,0 \% \Delta \Delta$ & $0 \% 10$ & $\widehat{H D}$ & 100 & \\
\hline$r, N \in G 9$ & 1,0rTs & $0,0 Y A V$ & $0,00 \pi r$ & $-1,1091$ & $-1, \pi Y \mid 9$ & $-0,1099$ & $-0,0 F_{0} \mid$ & $\widehat{J D}$ & & \\
\hline 00944 & $\% \Delta 9 V$ & op rar & וגו1 & $0,1 \times 91$ & $0,1 \% \circ$ & $0,1 \mathrm{frr}$ & $0,10 A Y$ & $\widehat{K L D}$ & & \\
\hline$\%$ \%1 & \% TIF & 0,0191 & 00100 & $0,19 \%$ & ०N०ᄉr & $0,0 \wedge Y 4$ & 0,0991 & $\widehat{H D}$ & ro & \\
\hline $1,910 \mathrm{r}$ & OKOFY & $0,0 \Delta Q F$ & OOTr & $-1,9 \circ 49$ & $-0 / \Delta \circ V V$ & $-0,191$ & $-0,0 \wedge V r$ & $\widehat{J D}$ & & \\
\hline \% & $0,0 \times 90$ & $0,0 \% \pi$ & $0,01 \mathrm{VY}$ & $0 \% A \Delta A$ & $0,094 \pi$ & 0,0979 & $0,10 \%$ & $\widehat{K L D}$ & & تى \\
\hline \%Orद & OOTIT & $0,01 T \Lambda$ & $0,0 \% 44$ & $0, V A r$ & 0,0911 & $0,0 \mathrm{VAM}$ & $0,0<91$ & $\widehat{H D}$ & $\Delta_{0}$ & \\
\hline 1,4194 & 0 TYYA & 0,0 YAT & 00104 & $-1, \pi 914$ & $-0,4490$ & $-0,09 \pi T$ & $-0001 T$ & $\bar{J} \bar{D}$ & & \\
\hline$\% 100$ & $0,014 \Lambda$ & 0,0019 & 00090 & $0,0 Y Y Y$ & $0 \% \forall \Delta Y$ & $0 \% 00 \wedge$ & $00 \mathrm{OHq}$ & $\widehat{K L D}$ & & \\
\hline oOYOY & 0,191 & 0,0109 & 00049 & 0,0994 & 0,0901 & o, KYYY & $\%$ \% Qq & $\widehat{H D}$ & 100 & \\
\hline 1,4940 & $0,1 \Lambda_{0} 1$ & 0,0491 & $000 \Delta 9$ & $-1,0199$ & $-0, \pi 00 \wedge$ & $-0,0 \Delta V I$ & $-0,0 \mu r \mu$ & $\widehat{J D}$ & & \\
\hline$\% \Delta V I$ & $0 \% \times \wedge 9$ & 0,0 Y & $\% 0$ \% & V & O & 90,100 & $0,10 \mathrm{Vr}$ & $\widehat{K L D}$ & & \\
\hline$\% 0499$ & 0,0111 & 0,0149 & $\% \circ \mathrm{Vr}$ & $0, N Y 4$ & 0,0914 & 0,0949 & $\% 0$ OTD & $\widehat{H D}$ & $r_{0}$ & \\
\hline$r / 010 r$ & $0,940 \wedge$ & $\%$ prs & 00191 & - & - ONGMY & $-0,1 T \Delta \Delta$ & $-0,0 \mathrm{VQV}$ & $\widehat{J D}$ & & \\
\hline$\% \circ+91$ & $00 r+9$ & $0,0 Y 09$ & 00190 & $0, \wedge \wedge F$ & 0,094 & 0,1000 & $0,10 \mathrm{VD}$ & $\widehat{K L D}$ & & تى $(\nu=10)$ \\
\hline \%०Y & 0,0110 & $\% \circ \circ \wedge \Delta$ & 0,0049 & $0,0 \Lambda \mathrm{V}$ & $0,0 \wedge \vee$ & o,هVA & 0,0494 & $\overline{H D}$ & $\Delta^{\circ}$ & \\
\hline TMIOS & $\circ \Delta \wedge \mid V$ & orTYY & 0,0091 & $-r, 10 k 9$ & $-O N T V T$ & -0, & $-0,0 \Delta T V$ & $\widehat{J D}$ & & \\
\hline ryוtro & 0,0109 & $0,0 \circ \wedge 1$ & $0 \% \circ \Delta \wedge$ & 0,0 Yor & ००ळ०Y & $0,0 \Delta 91$ & $0,090 \%$ & $\widehat{K L D}$ & & \\
\hline$\%$ \% KYq & $0,0 Y 19$ & $0,0 \circ \wedge 9$ & poOrt & $0,0 Y M A$ & $0,0 \wedge \circ Y$ & $0 \% 4 \circ V$ & 0,0490 & $\widehat{H D}$ & 100 & \\
\hline T,TIGY & 0, KTYY & $0 O Y Y D$ & 0,0449 & $-|, /| q \mu$ & $-\circ \Delta Q 9 Y$ & $-0,0 M M 9$ & $-0,0 Y 10$ & $\overline{J D}$ & & \\
\hline
\end{tabular}

براى توابع مفصل در نظر گرفته شده، دقت بيشترى در مقايسه با اندازه وابستخى كولبك-ليبلر و جفرى

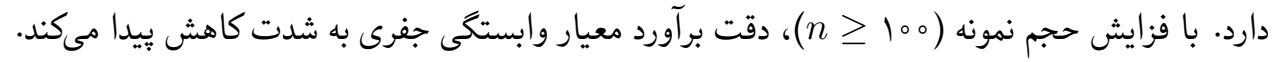

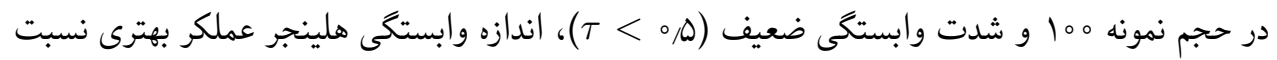

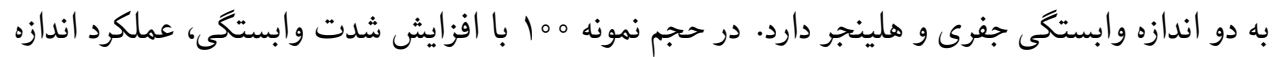

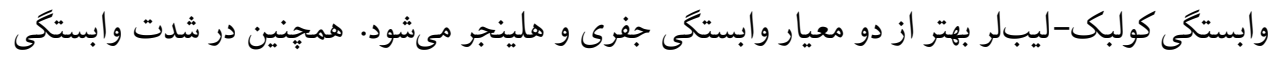
خيلى ضعيف (r آه 


\section{4 كاربرد در هيدرولوزى}

تحليل آمارى خشكسالى يكى از نيازهاى مهم هوا و اقليم شناسى، هيدرولوزى و سيستمهاى برنامهريزى و

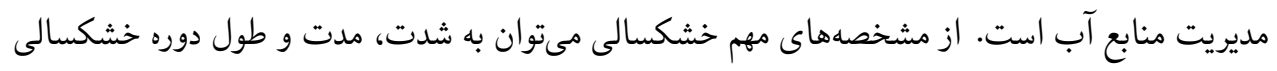

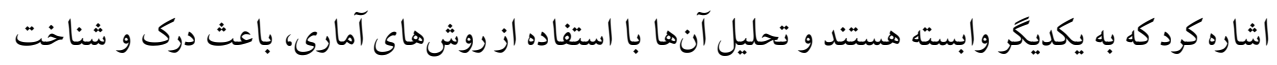

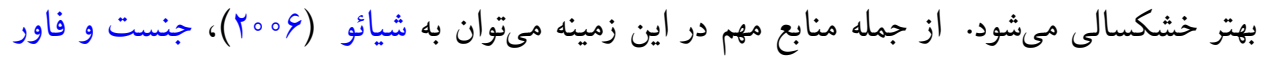
(Y०V)

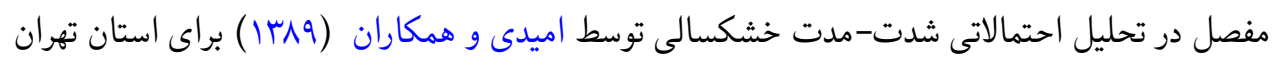

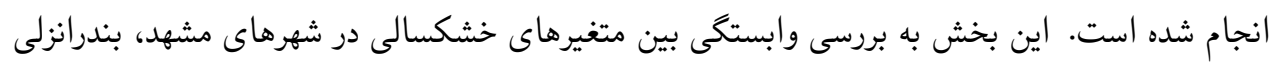

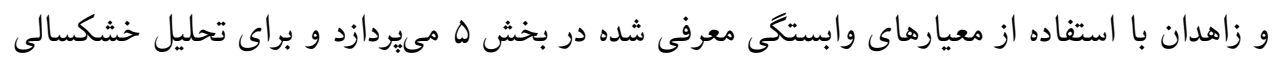

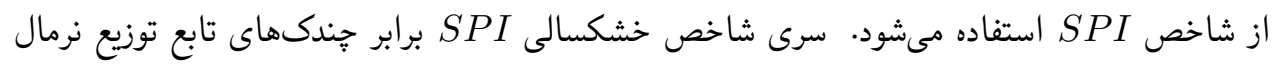

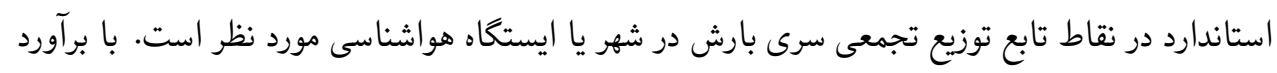

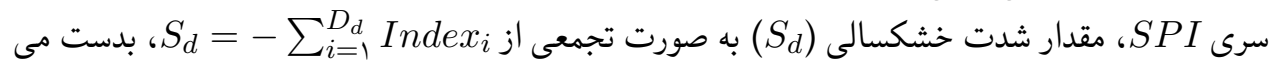

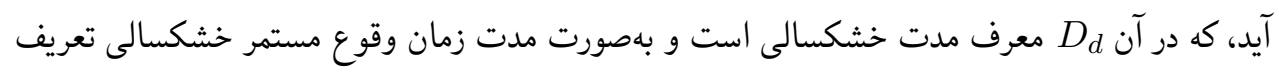
مىشود و همجنين SPI

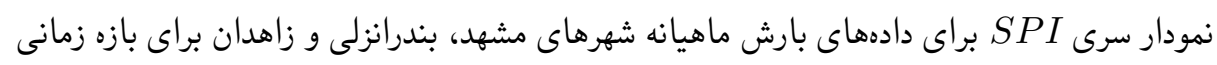

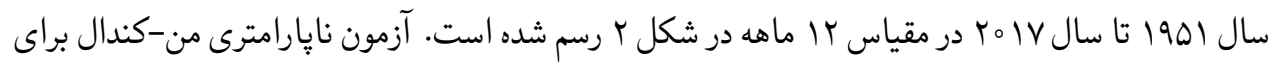

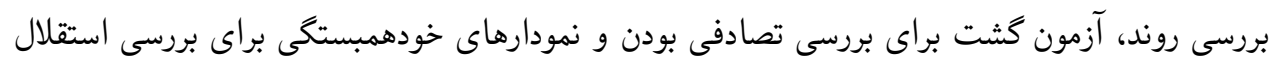

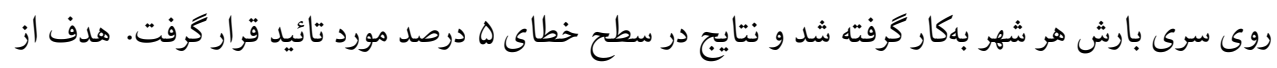

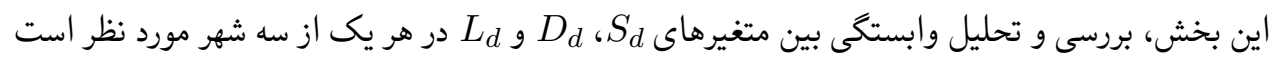

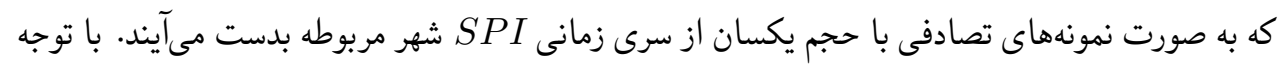

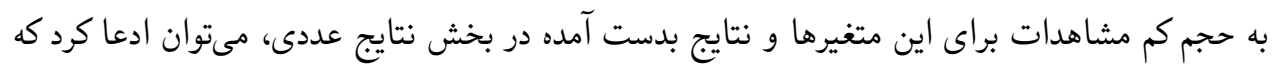

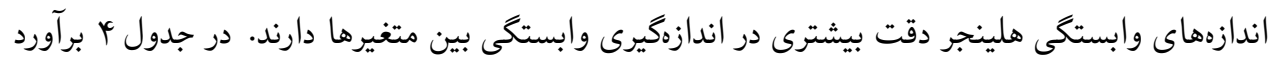

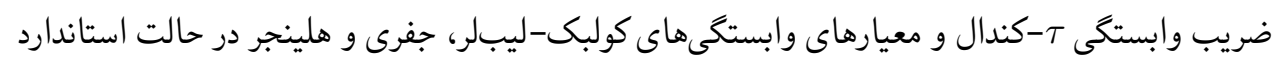

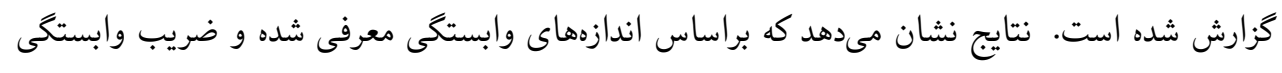

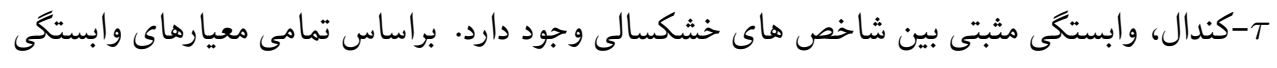

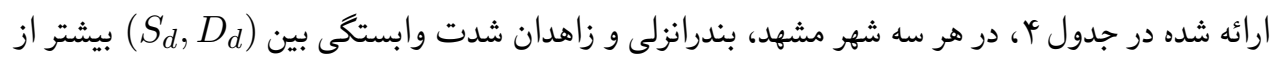




$$
\text { شدت وابستخى بين ساير متغيرها است. }
$$
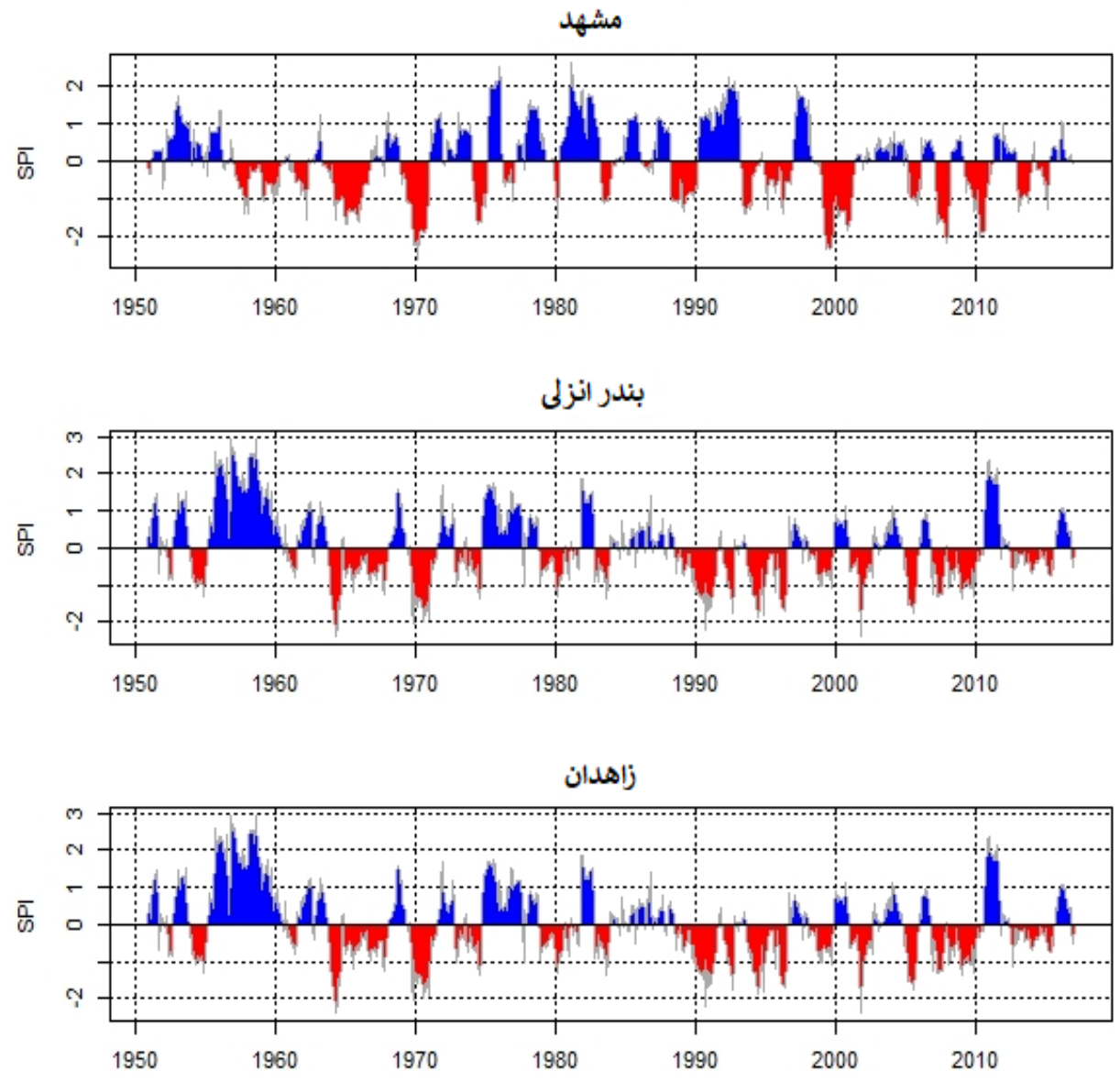

شكل r. سرى SPI براى دادهاى بارش ماهيانه شهرهاى مشهد، بندرانزلى و زاهدان

\section{بحث و نتيجهکيرى}

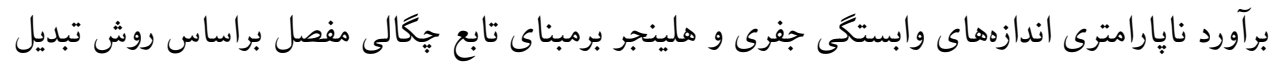

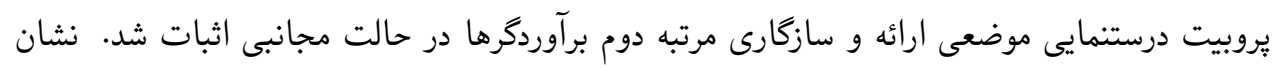

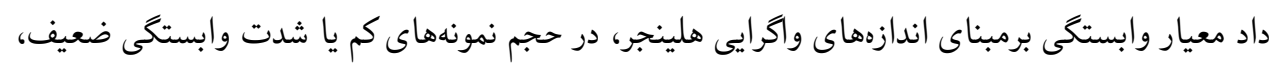




\begin{tabular}{|c|c|c|c|c|}
\hline$\left(D_{d}, L_{d}\right)$ & $\left(S_{d}, L_{d}\right)$ & $\left(S_{d}, D_{d}\right)$ & وابستخى & شهر \\
\hline 0,4549 & o/DTH & $0, V V I Y$ & $\hat{\tau}$ & \\
\hline $0 / \Delta \Delta \psi \mid$ & $0,949 V$ & 0,1499 & $\widehat{K L D}^{*}(c)$ & مشهد \\
\hline - ptsad & $.0 \mathrm{kqr}$ & $0 N T \circ 1$ & $\widehat{J D}^{*}(c)$ & $(n=r \varphi)$ \\
\hline $0, r \Delta \circ \Delta$ & OMTYO & 0,4091 & $\widehat{H D}^{*}(c)$ & \\
\hline oOHYA & $0 / 0941$ & $0,9 \wedge \vee 1$ & $\hat{\tau}$ & \\
\hline 0,1991 & $0,9 V I r$ & ONKTO & $\widehat{K L D}^{*}(c)$ & بندرانزلي \\
\hline $0,90 \Delta V$ & $0 / \Delta \Lambda \mu r$ & ONOTV & $\widehat{J D}^{*}(c)$ & $(n=k x)$ \\
\hline $0,4 \circ 41$ & OMYAT & optyat & $\widehat{H D}^{*}(c)$ & \\
\hline 0 opqYT & ODYTV & -NMKT & $\hat{\tau}$ & \\
\hline $0,9 Y 11$ & $0,94 \circ 9$ & $0,90 \wedge r$ & $\widehat{K L D}^{*}(c)$ & زاهدان \\
\hline $0 / \Delta \Lambda F \Lambda$ & $0, g \mid \Delta Y$ & $\circ \wedge \Delta V I$ & $\widehat{J D}^{*}(c)$ & $(n=r r)$ \\
\hline o & 0 M MOV & $0 / 09 G Y$ & $\widehat{H D}^{*}(c)$ & \\
\hline
\end{tabular}

بسيار دقيقتر از اندازه وابستخى كولبك-ليبلر عمل مىكند. همجنين در شدتهاى وابستكى مختلف و

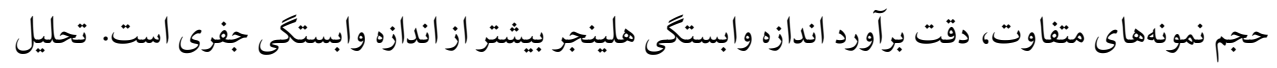

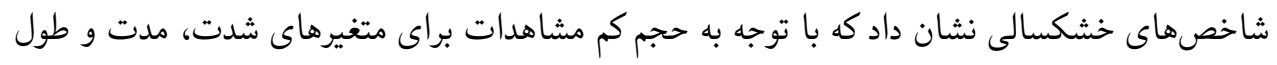

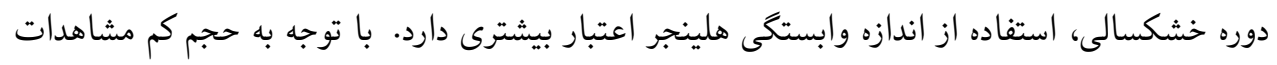

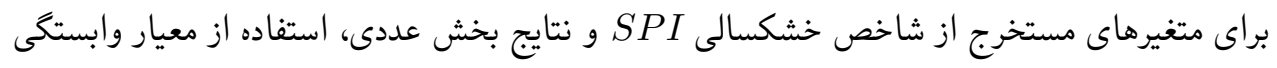

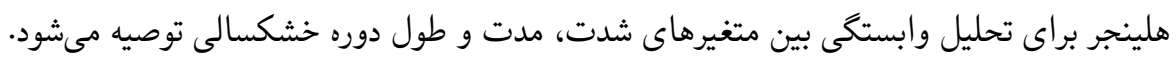

\section{تقدير و تشكر}

نويسندكان از بيشنهادات ارزنده داوران گرامى، هيئت تحريريه و ويراستار محترم مجله كه باعث بهبود و ارائه بهتر اين مقاله گرديد، كمال تشكر و قدردانى را دارند. 


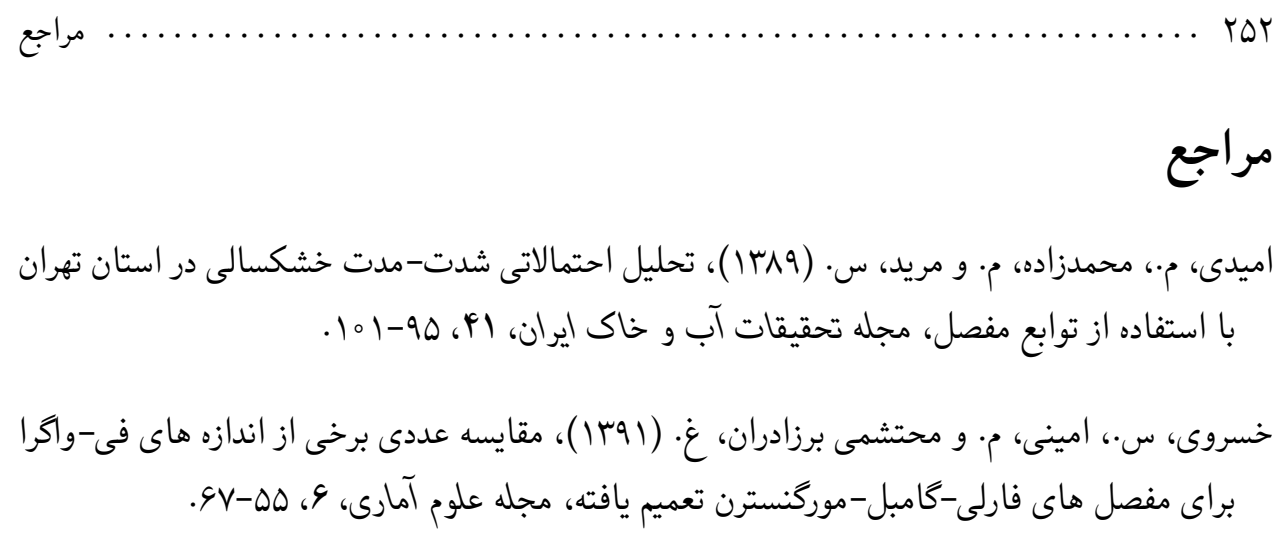

Ahmad, I., and Lin, P. E. (1976), A Nonparametric Estimation of the Entropy for Absolutely Continuous Distributions, IEEE Transactions on Information Theory, 22, 372-375.

Belalia, M., Bouezmarni, T., Lemyre, F. C., and Taamouti, A. (2017), Testing Independence Based on Bernstein Empirical Copula and Copula Density, Journal of Nonparametric Statistics, 29, 346-380.

Blum, J. R., Kiefer, J., and Rosenblatt, M. (1961), Distribution Free Tests of Independence Based on the Sample Distribution Function, The Annals of Mathematical Statistics, 32, 485-498.

Blumentritt, T., and Schmid, F. (2012), Mutual Information as A Measure of Multivariate Association: Analytical Properties and Statistical Estimation, Journal of Statistical Computation and Simulation, 82, 1257-1274.

Bouezmarni, T., El Gouch, A., and Taamouti, A. (2013), Bernstein Estimator for Unbounded Copula Densities, Statistics and Risk Modeling, 30, 343-360.

Charpentier, A., Fermanian, J., and Scaillet, O. (2006), Copulas: From theory to application in Finance, Chapter The Estimation of Copulas: Theory and Practice, Risk Books, Torquay, UK, 1, 35-62. 
ror

Chen, L., and Guo, S. (2019), Copulas and Its Application in Hydrology and Water Resources, Springer.

Geenens, G., Charpentier, A., and Paindaveine, D. (2017), Probit Transformation for Nonparametric Kernel Estimation of the Copula Density, Bernoulli, 23, 1848-1873.

Genest, C., and Favre, A. C. (2007), Everything You Always Wanted to Know About Copula Modeling but Were Afraid to Ask, Journal of Hydrologic Engineering, 12, 347-368.

Genest, C., Masiello, E., and Tribouley, K. (2009), Estimating Copula Densities Through Wavelets, Insurance: Mathematics and Economics, 44, 170-181.

Genest, C., and Remillard, B. (2004), Test of Independence and Randomness Based on the Empirical Copula Process, Test, 13, 335-369.

Joe, H. (1989), Relative Entropy Measures of Multivariate Dependence, Journal of the American Statistical Association, 84, 157-164.

Joe, H. (2014), Dependence Modeling with Copulas, Chapman and Hall/CRC.

Kauermann, G., Schellhase, C., \& Ruppert, D. (2013), Flexible Copula Density Estimation with Penalized Hierarchical B-Splines, Scandinavian Journal of Statistics, 40, 685-705.

Kullback, S., and Leibler, R. A. (1951), On Information and Sufficiency, The Annals of Mathematical Statistics, 22, 79-86.

Loader, C. R. (1996), Local Likelihood Density Estimation, The Annals of Statistics, 24, 1602-1618. 
Ma, J., and Sun, Z. (2011), Mutual Information is Copula Entropy, Tsinghua Science \& Technology, 16, 51-54.

Micheas, A. C., and Zografos, K. (2006), Measuring Stochastic Dependence Using $\Phi$-Divergence, Journal of Multivariate Analysis, 97, 765-784.

Mohtashami Borzadaran, G. R. and Amini, M. (2010), Information Measures via Copula Functions, Journal of Statistical Research of Iran, 7, 47-60.

Nagler T (2014), Kernel Methods for Vine Copula Estimation, Master's Thesis, Technische Universität München.

Nagler, T. (2018), kdecopula: An R Package for the Kernel Estimation of Bivariate Copula Densities, Journal of Statistical Software, 84, 1-22.

Nelsen, R. B. (2007), An Introduction to Copulas, Springer Science and Business Media.

Shiau, J. T. (2006), Fitting Drought Duration and Severity with Twodimensional Copulas, Water Resources Management, 20, 795-815.

Sklar, M. (1959), Fonctions de Repartition an Dimensions et Leurs Marges, Publications de l'Institut de Statistique de l'Université de Paris, 8, 229-231.

Song, S., and Singh, V. P. (2010), Meta-Elliptical Copulas for Drought Frequency Analysis of Periodic Hydrologic Data, Stochastic Environmental Research and Risk Assessment, 24, 425-444.

Wen K and Wu X (2015), Transformation-Kernel Estimation of the Copula Density, Working Paper, http://agecon2.tamu.edu/people/faculty/ wu-ximing/agecon2/public/copula.pdf. 
Journal of Statistical Sciences, Spring and Summer, 2021

Vol. 15, No. 1, pp 233-254

DOI: $10.29252 /$ jss.15.1.233

\title{
Bivariate Dependency Analysis using Jeffrey and Hellinger Divergence Measures based on Copula Density Estimation by Improved Probit Transformation
}

Mohammadi, M., Emadi, M., Amini, M.

Department of Statistics, Ferdowsi University of Mashhad, Mashhad, Iran.

\begin{abstract}
Divergence measures can be considered as criteria for analyzing the dependency and can be rewritten based on the copula density function. In this paper, Jeffrey and Hellinger dependency criteria are estimated using the improved probit transformation method, and their asymptotic consistency is proved. In addition, a simulation study is performed to measure the accuracy of the estimators. The simulation results show that for low sample size or weak dependence, the Hellinger dependency criterion performs better than Kullback-Libeler and Jeffrey dependency criteria. Finally, the application of the studied methods in hydrology is presented.
\end{abstract}

Keywords: Divergence, dependency, copula density, probit transformation.

Mathematics Subject Classification (2010): 62G10, 62G07, 62H15. 